\title{
Electrical analogs of curved beams and application to piezoelectric network damping
}

\author{
Robin Darleux \\ Boris Lossouarn $1 D$ \\ Laboratoire de Mécanique des Structures et des Systèmes Couplés (LMSSC), \\ Conservatoire national des arts et métiers (Cnam), Paris, France
}

\author{
Ivan Giorgio \\ Francesco dell'Isola \\ Department of Civil, Construction-Architectural and Environmental Engineering (DICEAA) and \\ International Research Center on Mathematics and Mechanics of Complex Systems (M\&MoCS), \\ University of L'Aquila, Italy
}

\section{Jean-François Deü \\ Laboratoire de Mécanique des Structures et des Systèmes Couplés (LMSSC), Conservatoire national des arts et métiers (Cnam), Paris, France}

Received 4 December 2020; accepted: 2 June 2021

\begin{abstract}
In this paper, the method of electric analog synthesis is applied to design a piezo-electro-mechanical arch able to show the capacity of multimodal damping. An electric-analog circuit is designed by using a finite number of lumped elements representing the equivalent of a curved beam. Spatial and frequency coherence conditions are proven to be verified for the modes to be damped: in fact, lumped-element circuit can damp only a finite number of vibration modes. Analogous boundary conditions are ensured, so that natural frequencies and mode shapes of both the curved beam and the analog circuit are equal. The instance considered here is the vibration mitigation of a piezo-electro-mechanical arch. Having a view towards prototypical applications, all simulations consider values of physically feasible passive circuital elements. It is believed that the present results may represent a step towards the design of multi-physics metamaterials based on micro-structures exploiting the principle of multimodal damping.
\end{abstract}

\section{Keywords}

Electromechanical analogy, lumped-element model, piezoelectric coupling, vibration mitigation, multimodal damping 


\section{Introduction}

Only starting from the 1960 s, digital computing prevailed upon analog computing in engineering and scientific applications. Actually, for at least three decades it was not clear whether digital computers were effectively more efficient than analog computers, albeit it was clear that digital computers could, in principle, be used for every conceivable computation by means of suitable programming. Analog computers being specialized for a precise set of calculations seemed to have an unbridgeable advantage. However, in the late 1960s it became evident that the reprogramming capacities of digital computers made them the best choice in almost every application. Therefore, the use of the finite element method as a modeling tool for static or low-frequency dynamic problems quickly became the only conceived option. In fact, the rise of the finite element method coincides with the extension of digital computer processing power. Previously every design and simulation could be made only by means of analog computers, also in industrial applications. The research department in General Electric developed, under the influence of Gabriel Kron [1], important techniques for synthesizing analog circuits for a large class of ordinary differential equations (ODEs) and partial differential equations (PDEs) and applied them in a wide range of engineering and scientific problems. In particular, the pioneering work by Kron shows how the behavior of mechanical systems can be simulated by using electrical networks: these networks were designed to exhibit, after a suitable dimensional analysis, exactly the same static or dynamic behavior as their analog mechanical systems. In this way, it is possible to forecast the values of the mechanical variables of interest (such as displacements or forces) by measuring the analog electrical variables (currents or voltages) in the synthesized electric circuit. An extensive work about the techniques for designing electrical analogs of mechanical structures, such as beams [2-4], plates [2], and shells [5], started already in the early 1950s. Most of these works were considered obsolete once the finite element method became a global standard also for mechanical simulations. However, the concept of designing analog structures has been revived at the beginning of the 2000s for vibration damping purposes [6]. In [7], it is shown that, by means of piezo-electro-mechanical coupling with an analog circuit (having the same modal properties as the mechanical system), there is an efficient energy transduction in the whole vibrating structure. As a consequence, the principle of distributed piezoelectric circuit damping emerged [8-24]. In fact, vibration mitigation can be achieved by optimally choosing the electrical components so that damped electrical resonances are tuned to the targeted mechanical resonance.

One way to define electrical analogs is to specify an electromechanical analogy $[25,26]$. Piezoelectric network damping of beams [15-17] and plates $[18,19]$ were analyzed in the linear regime. These pioneer works were mainly based on the application of the forcecurrent and velocityvoltage analogy, also called indirect analogy by Bloch [25]. Instead, in the present work, the force-voltage and velocity-current analogy, also called direct electromechanical analogy, is applied. Indeed, this analogy allows for the representation of an electromechanical transducer of piezo-electro-mechanical coupled systems with passive electrical components when this transducer is based on electrostatic forces [25]. Direct analogy is thus the most convenient analogy to represent piezoelectric coupling with passive electrical components. Hence, one can derive a fully passive representation of a mechanical structure coupled to an electrical network via piezoelectric transducers. The resulting electrical analogs have been implemented recently for vibration mitigation of rods [20], beams [21], and plates [22, 27]. However, piezoelectric network damping of complex structures has barely been covered in the scientific literature. The next objective is thus to define analog electrical networks for structures which include curvatures, varying geometrical parameters or even anisotropic materials. A solution would be to define an electrical analog for each considered structure. However, it might become difficult to do so for any 3D body. Therefore, rather than defining complex analog networks, the idea is to create a library of basic elementary analogs. Different elements could be then assembled to find the analog of more complex structures. An example of this procedure is available in [3], where the dynamics of an air frame is replicated by an assembly of beam elements in bending and torsion. The present paper contributes to expend this library of electrical analogs with an original solution for the analog of a curved beam, which is then used in a problem of multimodal vibration damping by means of piezoelectric coupling.

The paper is organized as follows. Section 2 is concerned with the technique adopted to obtain an analogous electrical network from a mechanical structure. In Section 3, this method is applied to a curved beam composed of an arc of the circumference. In particular, we focus our attention on two formulations: one based on constitutive relationships with the same hypotheses as Euler-Bernoulli beams; the other is a Hencky-type formulation where the structure is discretized employing small straight elements able to describe a curved beam when assembled together. Based on these results, Section 4 is devoted to presenting an electric circuit that, coupled with the curved beam, can reduce its vibrations. Some conclusions are finally drawn in Section 5. 
Table I. Direct and indirect electromechanical analogies

\begin{tabular}{lll}
\hline \multirow{2}{*}{ Mechanical quantities } & Electrical quantities & \\
\cline { 2 - 3 } & Direct analogy & Indirect analogy \\
\hline Force $-F$ and moment $-M$ & Voltage $V$ & Electrical current $i$ \\
Linear velocity $\dot{u}$ and angular velocity $\dot{\theta}$ & Electrical current $i$ & Voltage $V$ \\
Displacement $u$ and angle $\theta$ & Electrical charge $q$ & Flux linkage $\int V \mathrm{~d} t$ \\
Compliance & Capacitance $C$ & Inductance $L$ \\
Mass and rotational inertia & Inductance $L$ & Capacitance $C$ \\
Viscous damping & Resistance $R$ & Conductance $1 / R$ \\
\hline
\end{tabular}

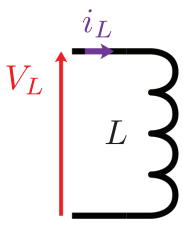

(a)

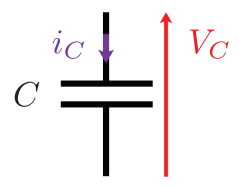

(b)

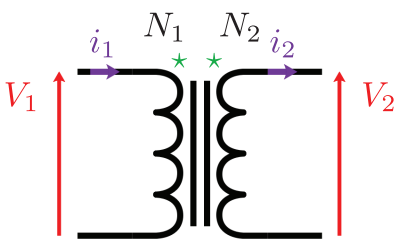

(c)

Figure I. Schematic representation of the four ideal purely passive electrical components: (a) an inductor, (b) a capacitor, and (c) a transformer (star symbols $\star$ inform about the winding direction).

\section{Method for defining an electrical analog of a mechanical structure}

\section{I. Finite difference scheme and electromechanical analogy}

In order to find a discrete electrical analog, the first step is to define a discrete model of the continuous mechanical structure. The choice of the discretization is important because it leads to different system of equations [28]. We choose to use the simplest grid so that the resulting number of components in the discrete unit cell is as low as possible. This is done by applying a first-order finite difference pattern to the set of differential equations describing the mechanical structure. We consider the following finite difference expressions for any physical quantity $g$ :

$$
\left(\frac{\partial g}{\partial x}\right)_{\mathrm{I}}=\frac{g_{\mathrm{R}}-g_{\mathrm{L}}}{a}, \quad\left(\frac{\partial g}{\partial x}\right)_{\mathrm{L}}=\frac{g_{\mathrm{I}}-g_{\mathrm{L}}}{a / 2} \quad \text { and } \quad\left(\frac{\partial g}{\partial x}\right)_{\mathrm{R}}=\frac{g_{\mathrm{R}}-g_{\mathrm{I}}}{a / 2}
$$

where $a$ is the discretization step between the left and the right sides, denoted $\mathrm{L}$ and $\mathrm{R}$, and I corresponds to the internal equidistant position.

An electromechanical analogy $[25,26]$ is then applied to the set of discrete mechanical equations. The indirect analogy, which states that velocities are analogous to voltages and forces are analogous to electrical currents, is considered in some works on analogues of mechanical structures $[2,16,18]$. This analogy is summed up in Table 1. It is particularly well suited to the study of electromagnetic systems, as it allows the passive representation of electromagnetic coupling [25]. In the present work, we consider the direct electromechanical analogy, which states that voltages and electrical currents are respectively analogous to forces and velocities. It is here preferred because it allows the fully passive representation of piezoelectric transducers [25]. Once the electrical equations are written, the mechanical structure and its electrical analog can both be represented by lumped-element electrical circuits.

As our objective is to find purely passive electrical analogs, only passive components can be part of the resulting electrical network. The three classical passive and non-dissipative components are sketched in their ideal versions in Figure 1. An inductor of inductance $L$ and a capacitor of capacitance $C$ relate the respective electrical currents $i_{L}$ and $i_{C}$ flowing through them to the voltages $V_{L}$ and $V_{C}$ at their terminals by first order time differential equations:

$$
V_{L}=L \frac{d i_{L}}{d t}, \quad i_{C}=C \frac{d V_{C}}{d t} .
$$

Furthermore, the transformer is a two-port circuit which has two windings of conductive wire: a primary one of $N_{1}$ turns and a secondary one of $N_{2}$ turns. With the electrical currents $i_{1}$ and $i_{2}$ and the voltages $V_{1}$ and $V_{2}$ 


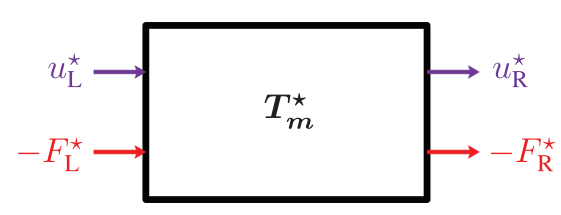

(a)

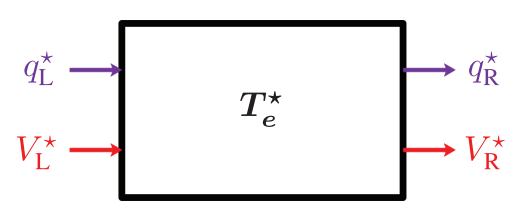

(b)

Figure 2. Schematic representation of (a) a mechanical unit cell and (b) an electrical unit cell of the corresponding discrete models.

pointing towards the respective terminals, a transformer of ratio $\hat{a}=N_{1} / N_{2}$ gives the following characteristic:

$$
\hat{a}=\frac{N_{1}}{N_{2}}=\frac{i_{2}}{i_{1}}=\frac{V_{1}}{V_{2}}
$$

\subsection{Spatial and frequency coherence conditions}

Several electrical unit cells need to be assembled to produce the analog of a multimodal mechanical structure. The assembled network is a multi-resonant system, whose modal properties can be modified by acting on its boundary conditions and on its components characteristics. Therefore, conditions should be applied to ensure that the network exhibits the desired dynamics.

First, the electrical network should exhibit the same mode shapes as the mechanical structure. To meet this condition, the mechanical boundary conditions should be adequately reproduced in the electrical network. Furthermore, the electrical analog has to be derived from a sufficiently refined discrete mechanical model which approximates the dynamic behavior of the continuous structure. A requirement is to adapt the structure discretization to the frequency range over which the analogy should be validated. A first criterion to meet a spatial coherence condition can be to consider at least 10 elements per wavelength:

$$
\frac{n_{e l m}}{N_{\max }} \geq 10
$$

where $n_{e l m}$ is the number of elements and $N_{\max }$ is the maximum number of wavelengths among the modes of the considered frequency range. This criterion can be part of the design process: once the frequency range is fixed, the natural frequencies and mode shapes can be predicted. By forecasting an approximation of $N_{\max }$, the number of electrical unit cells $n_{\text {elm }}$ can be deduced.

Second, if the spatial coherence condition is met, it is also required that the electrical network and the mechanical structure share the same natural frequencies. This frequency coherence condition is equivalent to ensure that they exhibit the same wave propagation properties.

The derivation of this condition is based on the 1D unit cells representations in Figure 2. The notation $u$, $F, q$, and $V$ denote displacements, mechanical loads, electrical charges, and voltages, respectively, whereas the $\mathrm{L}$ and $\mathrm{R}$ subscripts signal the left and right positions of the unit cells. Finally, the $\star$ superscript indicates non-dimensionalized quantities. The mechanical and electrical transfer matrices $T_{m}^{\star}$ and $T_{e}^{\star}$ of the discretized systems relate quantities on the left-hand side to quantities on the right-hand side of the unit cells:

$$
\left(\begin{array}{c}
u_{\mathrm{R}}^{\star} \\
-F_{\mathrm{R}}^{\star}
\end{array}\right)=T_{m}^{\star}\left(\begin{array}{c}
u_{\mathrm{L}}^{\star} \\
-F_{\mathrm{L}}^{\star}
\end{array}\right), \quad\left(\begin{array}{c}
q_{\mathrm{R}}^{\star} \\
V_{\mathrm{R}}^{\star}
\end{array}\right)=T_{e}^{\star}\left(\begin{array}{c}
q_{\mathrm{L}}^{\star} \\
V_{\mathrm{L}}^{\star}
\end{array}\right) .
$$

Consequently, the frequency coherence condition is met when $T_{e}^{\star}=T_{m}^{\star}$. Both systems would then have similar wave propagation properties, and thus equal natural frequencies if exhibiting analogous boundary conditions.

\subsection{From unit cells to a global model}

To define the full electrical analog of a mechanical structure, several unit cells should be assembled. As stated previously, the more cells used to discretize the network, the wider the frequency range over which the analogy is valid. To model the behavior of the assembled network, we propose to assemble elementary matrices just like in the finite element method. These matrices should relate external voltages to electrical charges, such as 
prescribed by the direct electromechanical analogy in Table 1. Using the notation of Equation (5), the electrical transfer matrix $T_{e}^{\star}$ can be separated into four square submatrices:

$$
T_{e}^{\star}=\left(\begin{array}{c|c}
A & B \\
\hline C & D
\end{array}\right)
$$

so that

$$
q_{\mathrm{R}}^{\star}=A q_{\mathrm{L}}^{\star}+B V_{\mathrm{L}}^{\star}, \quad V_{\mathrm{R}}^{\star}=C q_{\mathrm{L}}^{\star}+D V_{\mathrm{L}}^{\star} .
$$

The objective is to derive the elementary dynamic matrix $D_{e}^{\star}$, which is defined by

$$
\left(\begin{array}{c}
V_{\mathrm{L}}^{\star} \\
-V_{\mathrm{R}}^{\star}
\end{array}\right)=D_{e}^{\star}\left(\begin{array}{c}
q_{\mathrm{L}}^{\star} \\
q_{\mathrm{R}}^{\star}
\end{array}\right)
$$

Starting from Equation (7), $D_{e}^{\star}$ can be identified as

$$
D_{e}^{\star}=\left(\begin{array}{c|c}
-B^{-1} A & B^{-1} \\
\hline D B^{-1} A-C & -D B^{-1}
\end{array}\right)
$$

Note that the dynamic matrix is defined only if $B$ is invertible. This condition can be interpreted from Equation (7), in which setting $q_{\mathrm{L}}^{\star}=0$ for comprehension purposes does not restrain the problem: if $B$ is invertible, then $V_{\mathrm{L}}^{\star}$ can be calculated once the electrical charges $q_{\mathrm{R}}^{\star}$ are set. In a more general sense, this means that imposing the boundary electrical charges of the unit cell allows deriving the boundary voltages. From a mechanical point of view, this corresponds to being able to determine the boundary mechanical loads when the displacements are prescribed. If the discrete model is ill-defined, that is to say if $B$ is singular, then some numerical parameter must be added to enable further simulations.

Note that the dynamic matrix $D_{e}^{\star}$ relating non-dimensionalized voltages to non-dimensionalized electrical charges is not the required matrix going forward. The needed matrix is denoted $D_{e}$, and relates dimensionalized quantities:

$$
\left(\begin{array}{c}
V_{\mathrm{L}} \\
-V_{\mathrm{R}}
\end{array}\right)=D_{e}\left(\begin{array}{c}
q_{\mathrm{L}} \\
q_{\mathrm{R}}
\end{array}\right)
$$

If $B$ is invertible, then the dynamic matrix $D_{e}$ can be expressed, and so the elementary matrices of electrical "inertia" $M_{e l m}$ and of electrical "stiffness" $K_{e l m}$ can be extracted. The suggested method is to set the angular frequency $\Omega$ at zero to derive $K_{\text {elm }}$, and to calculate $M_{\text {elm }}$ afterwards:

$$
K_{e l m}=\left.D_{e}\right|_{\Omega=0} \quad \text { and } \quad M_{e l m}=\frac{K_{e l m}-D_{e}}{\Omega^{2}} .
$$

According to the direct electromechanical analogy in Table 1, $M_{\text {elm }}$ should depend on the inductive quantities of the unit cell, whereas $K_{\text {elm }}$ should depend on the capacitive quantities.

Then, following a geometric assembly process, the vectors $Q_{N}$ and $V_{N}$ which contain the values of electrical charges and external voltages in the entire network are related to each other by

$$
V_{N}=\left(K_{N}-\Omega^{2} M_{N}\right) Q_{N},
$$

with $M_{N}$ and $K_{N}$ being the assembled matrices of electrical "inertia" and electrical "stiffness," respectively.

\subsection{Analogy validation}

The designed electrical analog should have the same modal properties as the mechanical structure. These modal properties can be either extracted from experiments following an experimental modal analysis, or simulated. Simulations are the chosen path in this paper. Modal properties of the electrical network are obtained by solving the eigenvalue problem associated with Equation (12) in harmonic motion at angular frequency $\Omega$ :

$$
\left(K_{N}-\Omega^{2} M_{N}\right) Q_{N}=0 .
$$


Meanwhile, a 3D finite element model of a given mechanical structure is considered as a reference. The modes and natural frequencies of the structure are computed by resolving an eigenvalue problem that is analogous to that of Equation (13):

$$
\left(K_{m}-\Omega^{2} M_{m}\right) U=0
$$

where $K_{m}$ is the global stiffness matrix, $M_{m}$ is the global mass matrix, and $U$ is the vector of nodal displacements.

Conducting the analogy validation requires checking whether the frequency coherence condition is met. This is done by a direct quantitative comparison of electrical and mechanical natural frequencies. In the meantime, the analogy between the mechanical structure and the electrical network is validated if the spatial coherence condition is met. To this end, we propose to involve the modal assurance criterion (MAC) [29]. For any two eigenvectors $\Phi_{1}$ and $\Phi_{2}$ of same length:

$$
\operatorname{MAC}\left(\Phi_{1}, \Phi_{2}\right)=\frac{\left|\Phi_{1} \cdot \bar{\Phi}_{2}\right|^{2}}{\left(\Phi_{1} \cdot \bar{\Phi}_{1}\right)\left(\Phi_{2} \cdot \bar{\Phi}_{2}\right)}
$$

In this expression, $\bar{\Phi}$ is the conjugate vector of the eigenvector $\Phi$. This criterion indicates if the tested mode shapes look alike. If the MAC matrix contains values close to 1 , this means that the associated mode shapes are similar. On the other hand, low values in the MAC matrix are attained for two nearly orthogonal mode shapes.

\section{Curved beam electrical analog}

\section{I. Model A: from the constitutive equations}

Literature is abundant on the analytic study of curved structures, such as beams, rings, and arches [30]. In the case of an elongated structure, the simplest model applies the same hypotheses as the Euler-Bernoulli beam theory. Hence, we study the motion of a curved beam of mass density $\rho$, cross-section $S$, and radius of curvature $R$. Displacements amplitudes are denoted $U_{v}$ in the direction which is parallel to the neutral axis, and $U_{w}$ in the direction which is orthogonal to the neutral axis. All quantities depend on the arc length $s$. One can show that the curved beam dynamics in harmonic motion at angular frequency $\Omega$ is ruled by the following set of differential equations:

$$
-\rho S \Omega^{2} U_{v}=\frac{d N}{d s}+\frac{Q}{R} \quad \text { and } \quad-\rho S \Omega^{2} U_{w}=\frac{d Q}{d s}-\frac{N}{R}
$$

where $N$ and $Q$ denote the normal and shear forces in the beam, respectively. Thus, constitutive equations are required to completely define the structure behavior. One of them is the relation between the bending moment $M$ and the shear force $Q$ when neglecting the rotational inertia, which is a classical hypothesis of the EulerBernoulli beam theory. In this theory, shear deformations are neglected as well. As a consequence, the slope $\theta$ of the beam is related to the displacements. Finally, assuming a linear elastic material, the constitutive equations of the medium represented in Figure 3 relate the normal force $N$ and the bending moment $M$ to the displacements. These hypotheses create a set of four equations which, combined with the dynamic behavior described by Equation (16), form a set of first-order differential equations:

$$
\begin{aligned}
Q & =-\frac{d M}{d s}, & \theta & =\frac{d U_{w}}{d s}-\frac{U_{v}}{R}, \\
M & =E I \frac{d \theta}{d s}, & N & =E S\left(\frac{d U_{v}}{d s}+\frac{U_{w}}{R}\right) .
\end{aligned}
$$




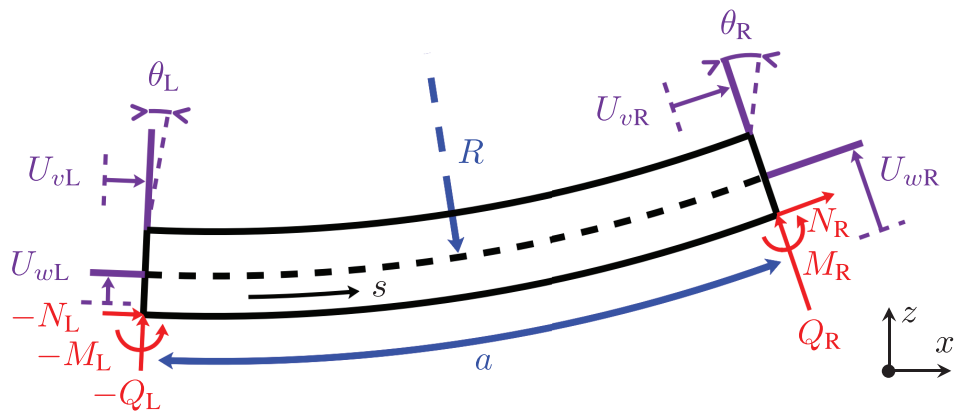

Figure 3. Continuous curved beam segment.

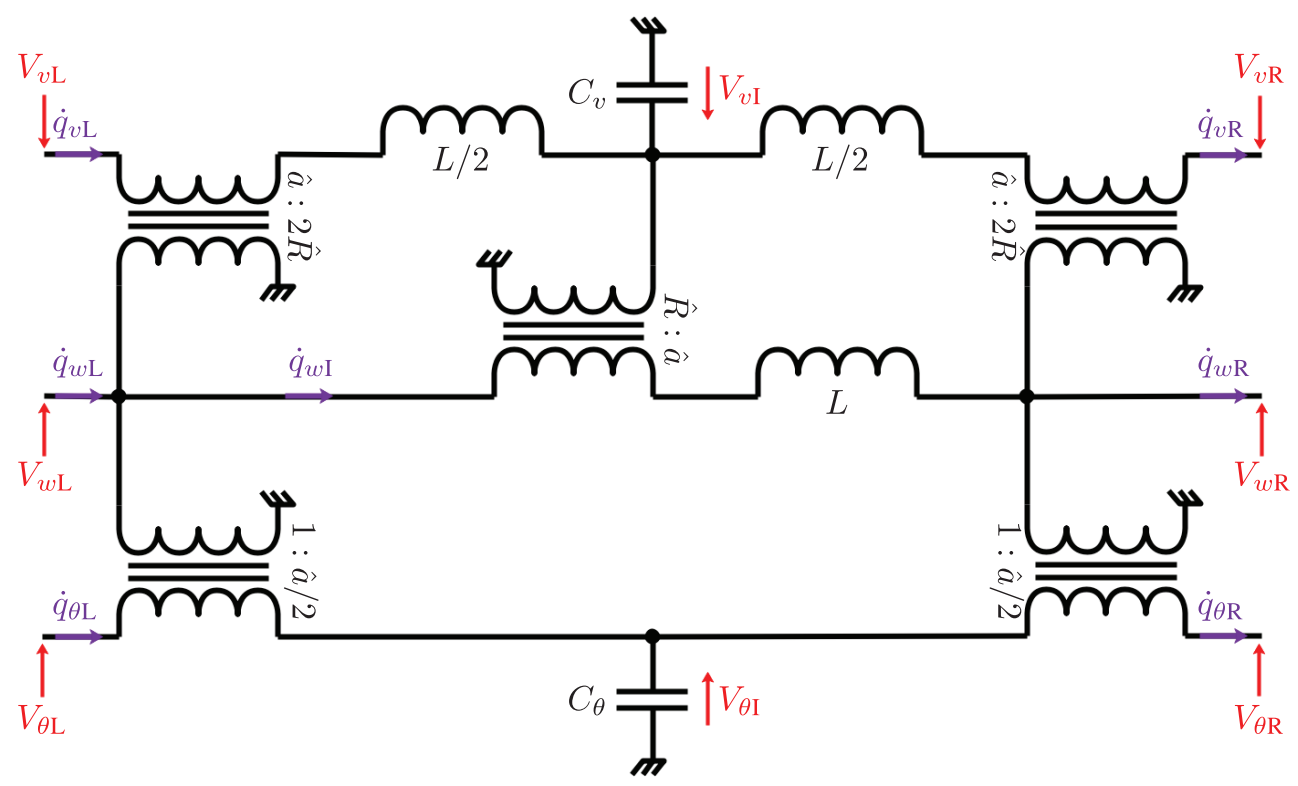

Figure 4. Unit cell for Model A of the curved beam electrical analog, which is defined by Equation (19).

Applying the finite difference scheme presented in Equation (1) to Equation (17) leads to a discrete model of the curved beam:

$$
\begin{aligned}
-\frac{m}{2} \Omega^{2} U_{v \mathrm{~L}} & =N_{\mathrm{I}}-N_{\mathrm{L}}+\frac{a}{2 R} Q_{\mathrm{L},} & & \frac{a}{2} \theta_{\mathrm{L}}=U_{w \mathrm{I}}-U_{w \mathrm{~L}}-\frac{a}{2 R} U_{v \mathrm{~L}}, \\
-\frac{m}{2} \Omega^{2} U_{v \mathrm{R}} & =N_{\mathrm{R}}-N_{\mathrm{I}}+\frac{a}{2 R} Q_{\mathrm{R}}, & & \frac{a}{2} \theta_{\mathrm{R}}=U_{w \mathrm{R}}-U_{w \mathrm{I}}-\frac{a}{2 R} U_{v \mathrm{R}}, \\
-m \Omega^{2} U_{w \mathrm{I}} & =Q_{\mathrm{R}}-Q_{\mathrm{L}}-\frac{a}{R} N_{\mathrm{I}}, & & \frac{a}{2} Q_{\mathrm{L}}=M_{\mathrm{L}}-M_{\mathrm{I}}, \\
N_{\mathrm{I}} & =K_{v}\left(U_{v \mathrm{R}}-U_{v \mathrm{~L}}+\frac{a}{R} U_{w \mathrm{I}}\right), & & \frac{a}{2} Q_{\mathrm{R}}=M_{\mathrm{I}}-M_{\mathrm{R}}, \\
M_{\mathrm{I}} & =K_{\theta}\left(\theta_{\mathrm{R}}-\theta_{\mathrm{L}}\right), & &
\end{aligned}
$$

with $m=\rho S a$ the mass of the beam segment, $K_{v}=E S / a$ its longitudinal stiffness, and $K_{\theta}=E I / a$ its bending stiffness.

The electrical analog of the curved beam is derived by replacing mechanical quantities by electrical quantities according to the direct electromechanical analogy summed up in Table 1. The resulting electrical unit cell 
Table 2. Curved beam analogous boundary conditions for a boundary on the right-hand side of the unit cell

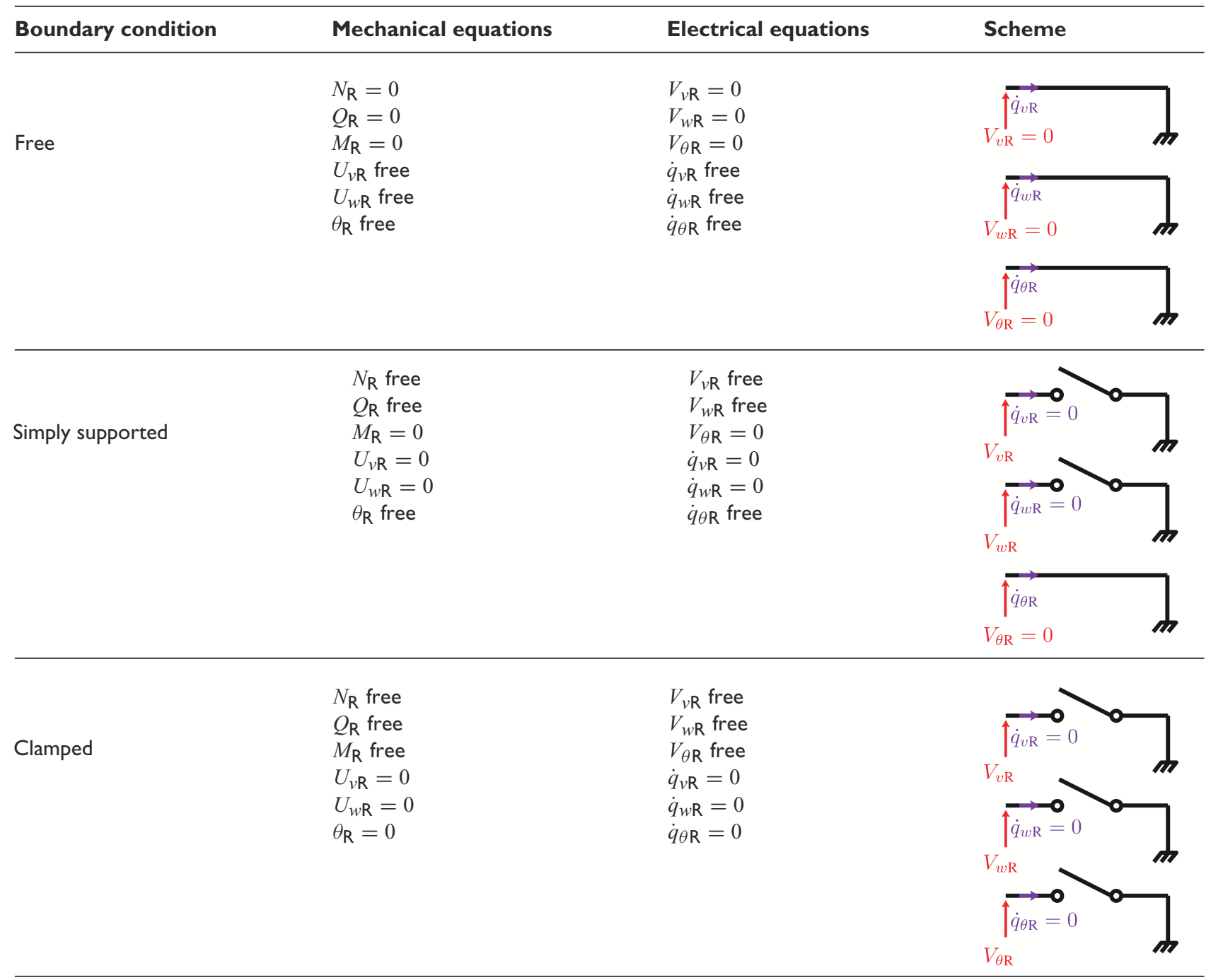

shown in Figure 4 is characterized by the following equations that directly come from Equation (18):

$$
\begin{aligned}
-\frac{L}{2} \Omega^{2} q_{v \mathrm{~L}} & =V_{v \mathrm{~L}}-V_{v \mathrm{I}}-\frac{\hat{a}}{2 \hat{R}} V_{w \mathrm{~L}}, & & \frac{\hat{a}}{2} q_{\theta \mathrm{L}}=q_{w \mathrm{I}}-q_{w \mathrm{~L}}-\frac{\hat{a}}{2 \hat{R}} q_{v \mathrm{~L}}, \\
-\frac{L}{2} \Omega^{2} q_{v \mathrm{R}} & =V_{v \mathrm{I}}-V_{v \mathrm{R}}-\frac{a}{2 \hat{R}} V_{w \mathrm{R}}, & \frac{\hat{a}}{2} q_{\theta \mathrm{R}} & =q_{w \mathrm{R}}-q_{w \mathrm{I}}-\frac{a}{2 \hat{R}} q_{v \mathrm{R}}, \\
-L \Omega^{2} q_{w \mathrm{I}} & =V_{w \mathrm{~L}}-V_{w \mathrm{R}}+\frac{a}{\hat{R}} V_{v \mathrm{I}}, & \frac{\hat{a}}{2} V_{w \mathrm{~L}} & =V_{\theta \mathrm{L}}-V_{\theta \mathrm{I}}, \\
C_{v} V_{v \mathrm{I}} & =q_{v \mathrm{~L}}-q_{v \mathrm{R}}-\frac{\hat{a}}{\hat{R}} q_{w \mathrm{I}}, & \frac{\hat{a}}{2} V_{w \mathrm{R}} & =V_{\theta \mathrm{I}}-V_{\theta \mathrm{R}} . \\
C_{\theta} V_{\theta \mathrm{I}} & =q_{\theta \mathrm{L}}-q_{\theta \mathrm{R}} . & &
\end{aligned}
$$

3.I.I. Analogous boundary conditions In the example of a simply supported mechanical boundary, the displacements $U_{v}$ and $U_{w}$ are forced at zero whereas the slope $\theta$ is left unassigned. Moreover, at the boundary, the normal and shear forces $N$ and $Q$ are undefined whereas the bending moment $M$ is equal to zero. For the unit cell in Figure 4, this corresponds to a short-circuit electrical connection on the slope grid, and to an open-circuit electrical connection on the two remaining displacements grids.

Electrical analogs of free and clamped boundary conditions can also be easily deduced. The associated discrete equations and sketches are gathered in Table 2 in the case of the right-hand side of the unit cells being a boundary. 
3.I.2. Frequency coherence conditions The expressions of non-dimensionalized displacements are given by $U_{v}^{\star}=$ $U_{v} / a$ and $U_{w}^{\star}=U_{w} / a$, rotations by $\theta^{\star}=\theta$, normal forces by $N^{\star}=N /\left(a K_{v}\right)$, shear forces by $Q^{\star}=a Q / K_{\theta}$, and bending moments by $M^{\star}=M / K_{\theta}$. To derive the frequency coherence conditions, the quantities on the right-hand side of the mechanical unit cell in Figure 3 that has been discretized are related to the quantities on the left-hand side:

$$
\begin{gathered}
\left(\begin{array}{c}
U_{\nu \mathrm{R}}^{\star} \\
U_{w \mathrm{R}}^{\star} \\
\theta_{\mathrm{R}}^{\star} \\
-N_{\mathrm{R}}^{\star} \\
-Q_{\mathrm{R}}^{\star} \\
-M_{\mathrm{R}}^{\star}
\end{array}\right)=\left(\begin{array}{cccccc}
1-\frac{\delta^{2}+\beta}{2} & -\delta & \frac{-\delta}{2} & -1 & \frac{\delta \beta}{2 \gamma} & 0 \\
\delta\left(1-\frac{\delta^{2}+\beta}{4}\right) & 1-\frac{\delta^{2}}{2} & 1-\frac{\delta^{2}}{4} & \frac{-\delta}{2} & \frac{1}{4}\left(1+\frac{\beta \delta^{2}}{\gamma}\right) & \frac{-1}{2} \\
0 & 0 & 1 & 0 & \frac{1}{2} & -1 \\
\beta\left(1-\frac{3 \delta^{2}+\beta}{4}\right) & -\delta \beta & \frac{-\delta \beta}{2} & 1-\frac{\delta^{2}+\beta}{2} & \frac{\beta \delta}{\gamma}\left(\frac{\delta^{2}+\beta}{4}-1\right) & 0 \\
\delta \gamma & \gamma & \frac{\gamma}{2} & \frac{\delta \gamma}{\beta} & 1-\frac{\delta^{2}}{2} & 0 \\
\frac{-\delta \gamma}{2} & \frac{-\gamma}{2} & \frac{-\gamma}{4} & \frac{-\delta \gamma}{2 \beta} & \frac{\delta^{2}}{4}-1 & 1
\end{array}\right)\left(\begin{array}{c}
U_{\nu \mathrm{L}}^{\star} \\
U_{w \mathrm{~L}}^{\star} \\
\theta_{\mathrm{L}}^{\star} \\
-N_{\mathrm{L}}^{\star} \\
-Q_{\mathrm{L}}^{\star} \\
-M_{\mathrm{L}}^{\star}
\end{array}\right), \\
\text { with } \quad \delta=\frac{a}{R}, \quad \beta=\frac{m \Omega^{2}}{K_{v}} \\
\text { and }
\end{gathered}
$$

Thus, we deduce that the electrical circuit in Figure 4 would have identical bending wave propagation properties as the discrete mechanical model if they share the same ratios $\delta, \beta$, and $\gamma$. This means that the frequency coherence conditions are

$$
\frac{a}{R}=\frac{\hat{a}}{\hat{R}}, \quad \frac{K_{v}}{m}=\frac{1}{L C_{v}}, \quad \frac{1}{a^{2}} \frac{K_{\theta}}{m}=\frac{1}{\hat{a}^{2}} \frac{1}{L C_{\theta}} .
$$

The last two conditions are identical to those of the rod and beam electrical analogs, as proposed in [20,21]. The first condition concerns the ratio of the unit cell length $a$ over the curvature $R$. It represents the coupling between traction and bending in the curved structure, whose influence is modeled by the three transformers of ratio $\hat{a}: \hat{R}$ or $\hat{a}: 2 \hat{R}$ in Figure 4 .

3. I.3. Unit cells assembly From Equations (6) and (20), it is deduced that $B$ is a singular matrix. As a consequence, we cannot compute the electrical dynamic matrix $D_{e}$. The reason for this is the same as for the Euler-Bernoulli beam and the Kirchhoff-Love plate discrete models derived previously in [21, 22]: the system of Equation (19) is ill-defined and cannot be solved. To avoid singularity problems, we suggest adding capacitors of capacitance $C_{0}$ at both sides of the $\dot{q}_{\theta}$ branch in Figure 4. Integrating these components to the curved beam electrical analog described by Equation (19) leads to a more complicated transfer matrix. Though not fully detailed here, the new $B$ matrix is

$$
B=\left(\begin{array}{ccc}
-1 & \frac{\delta \beta}{2 \gamma} & \frac{\delta \epsilon}{4} \\
\frac{-\delta}{2} & \frac{1}{4}\left(1+\frac{\beta \delta^{2}}{\gamma}\right) & \frac{1}{2}\left(\frac{\epsilon \delta^{2}}{4}-\epsilon-1\right) \\
\frac{\delta \gamma \epsilon}{4 \beta} & \frac{1}{2}\left(1+\epsilon-\frac{\epsilon \delta^{2}}{4}\right) & -\left(1+\epsilon+\frac{\gamma \epsilon^{2}}{16}\right)
\end{array}\right) \quad \text { with } \epsilon=\frac{C_{0}}{C_{\theta}} .
$$

Note that setting $C_{0}=0$, i.e., $\epsilon=0$, in this new expression of $B$ leads to the previously identified singular matrix. One can show that the determinant of this new matrix is

$$
\operatorname{det}(B)=-\frac{\epsilon}{4}\left[1+\epsilon\left(1+\frac{\gamma}{16 \beta}\left(\delta^{2}-\gamma \beta\right)\right)\right] \approx-\frac{\epsilon}{4} \quad \text { if } \epsilon \ll 1 .
$$

As expected, the $C_{0}$ capacitance should be different from zero to avoid numerical singularity, but should be small enough so that it does not alter the dynamics of the system $\left(\epsilon=10^{-6}\right.$ is suitable for the following numerical examples).

Now that $B$ is not singular anymore, the dynamic matrix can be derived. Using the notation in Figure 4, the values of the electrical charges and the voltages in one unit cell of the network are denoted $q_{N}$ and $v_{N}$ :

$$
\begin{aligned}
q_{N} & =\left(\begin{array}{llllll}
q_{v \mathrm{~L}} & q_{w \mathrm{~L}} & q_{\theta \mathrm{L}} & q_{v \mathrm{R}} & q_{w \mathrm{R}} & q_{\theta \mathrm{R}}
\end{array}\right)^{\mathrm{T}}, \\
v_{N} & =\left(\begin{array}{lllllll}
V_{v \mathrm{~L}} & V_{w \mathrm{~L}} & V_{\theta \mathrm{L}} & -V_{v \mathrm{R}} & -V_{w \mathrm{R}} & -V_{\theta \mathrm{R}}
\end{array}\right)^{\mathrm{T}} .
\end{aligned}
$$




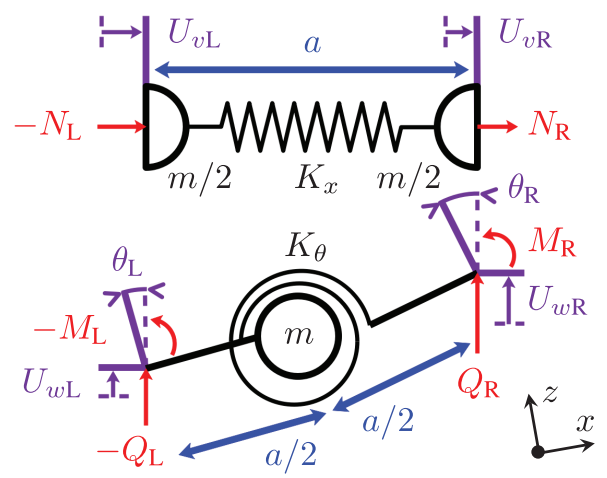

Figure 5. Uncoupled discrete model of a curved beam.

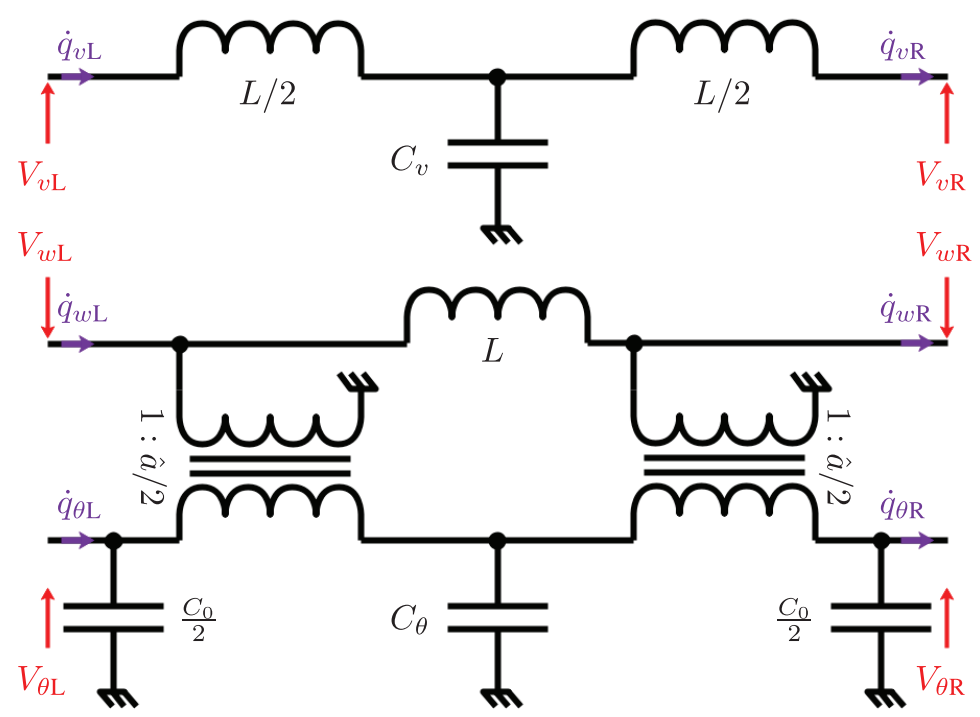

Figure 6. Uncoupled unit cell for Model B of the curved beam electrical analog.

Following the derivation of the dynamic matrix $D_{e}$, Equation (11) is applied to find the matrices $M_{e l m}$ and $K_{e l m}$. The resulting electrical elementary matrices are recorded in the Appendix. The elementary "inertia" matrix $M_{\text {elm }}$ is a function of the inductance value $L$, which is the electrical analogous quantity of the mass. The elementary "stiffness" matrix $K_{\text {elm }}$ is a function of the capacitances $C_{v}$ and $C_{\theta}$, which are the respective analogous quantities of the longitudinal and bending compliance. Then, following an assembly process, the vectors $Q_{N}$ and $V_{N}$ which contain the values of electrical charges and external voltages in the entire network are related to each other by

$$
V_{N}=\left(K_{N}-\Omega^{2} M_{N}\right) Q_{N}
$$

with $M_{N}$ and $K_{N}$ being the global matrices of electrical "inertia" and electrical "stiffness," respectively. After that, the mode shapes and natural frequencies of the electrical circuit can be estimated to be compared with those of the mechanical structure.

\subsection{Model B: from straight rod and beam elements}

If the structure is discretized enough, then a model of a curved beam can be seen as an assembly of straight elements with independent responses to longitudinal and bending loads, as seen in the discrete rod and beam models in Figure 5. Therefore, the electrical analog of such an element is defined by independently combining the rod electrical analog [20] and the beam electrical analog [21]. The resulting electrical analog is drawn in Figure 6. This electrical unit cell assumes that the structure is straight, and so that longitudinal and bending vibrations are not correlated. Figure 6 can thus be seen as the limit case of Figure 4 when the curvature tends 

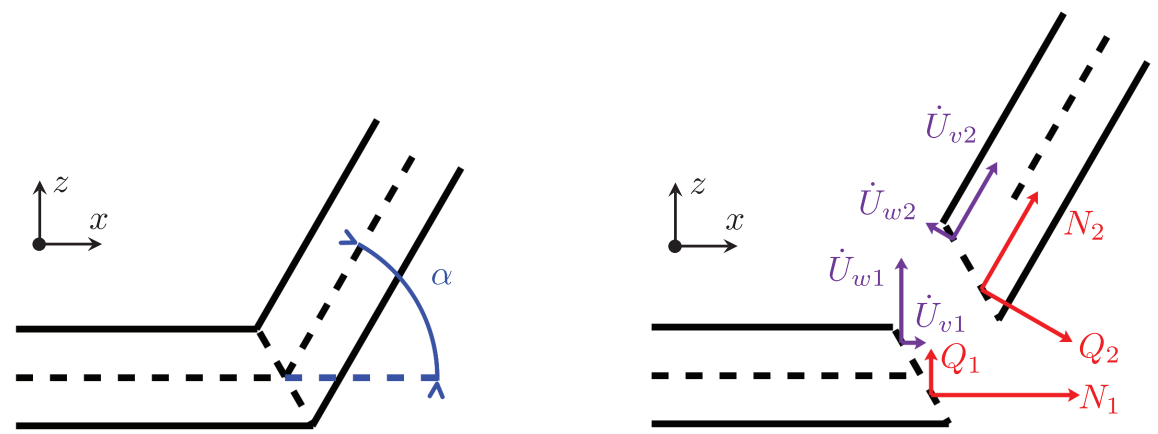

Figure 7. Transmission of loads and displacements through a tilted clamping.

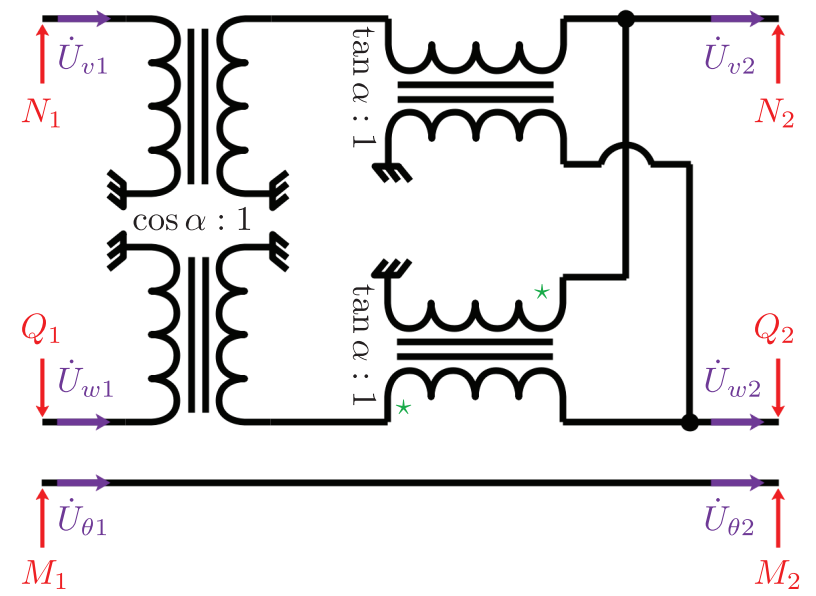

Figure 8. Electrical representation of the mechanical rotation in Equation (26).

to zero, i.e., the ratio $\hat{R} / \hat{a}$ tends to infinity. It is remarkable that the principle, on which the discretization just presented is based, has strong similarities to that exploited in Hencky-type discretization for beam structure [31-34].

To take the structure curvature into account, the elementary cells should be angularly offset according to the structure geometry. The clamped condition between the two straight segments with an angle $\alpha$ allows defining the transmission of loads and motion in both normal and transverse directions. Figure 7 highlights this concept. To electrically represent the angularly orientated link between two elements, an electrical analog of a rotation matrix should be designed. If " 1 " and " 2 " designate the left and right side of the link, respectively, then

$$
\left.\left.\left.\left.\begin{array}{l}
U_{v 2} \\
U_{w 2} \\
U_{\theta 2}
\end{array}\right)=\begin{array}{ccc}
\cos \alpha & \sin \alpha & 0 \\
-\sin \alpha & \cos \alpha & 0 \\
0 & 0 & 1
\end{array}\right)\left(\begin{array}{l}
U_{v 1} \\
U_{w 1} \\
U_{\theta 1}
\end{array}\right) \text { and } \begin{array}{l}
N_{2} \\
Q_{2} \\
M_{2}
\end{array}\right)=\begin{array}{ccc}
\cos \alpha & \sin \alpha & 0 \\
-\sin \alpha & \cos \alpha & 0 \\
0 & 0 & 1
\end{array}\right)\left(\begin{array}{l}
N_{1} \\
Q_{1} \\
M_{1}
\end{array}\right) .
$$

This set of equations can be passively represented in the form of an electrical circuit, in which the transformers ratios involve the $\alpha$ angle. This cell was first shown in [3], and is sketched in Figure 8. Finally, it is possible to define our Model B for the curved beam electrical analog. It is done by considering previously derived electrical circuits modeling longitudinal and bending wave propagation, and interconnecting them by electrical analogs of rotation matrices. The comparison between these two analogs is conducted in the following subsection.

Looking at the number of electrical components, the unit cell in Figure 6 contains three inductors, two capacitors, and two transformers. Moreover, Figure 8 shows that four transformers are required between two unit cells to make the rotation. This leads the full unit cell to three inductors, two capacitors, and six transformers. On the other hand, the unit cell in Figure 4 contains three inductors, two capacitors, and five transformers. However, the side transformers can be merged with the similar transformer of the adjacent cell. Each unit cell thus virtually contains three inductors, two capacitors, and three transformers. This difference in number of transformers highlights that the method of electrical analog derivation has a significant effect on the potential 


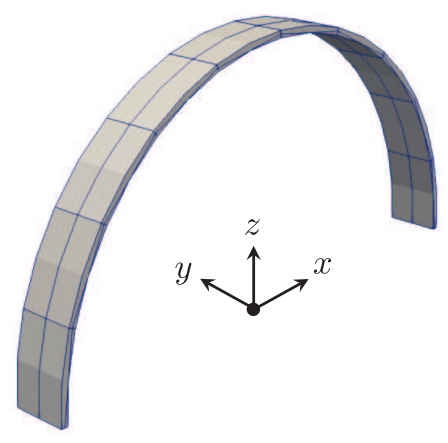

(a)

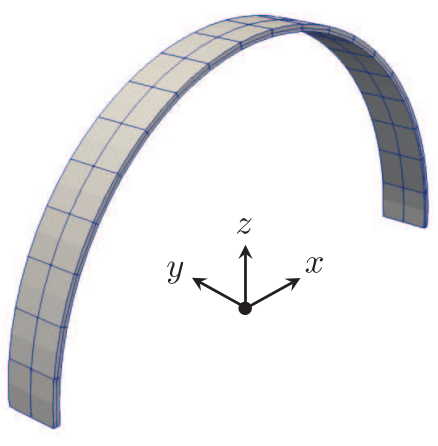

(b)

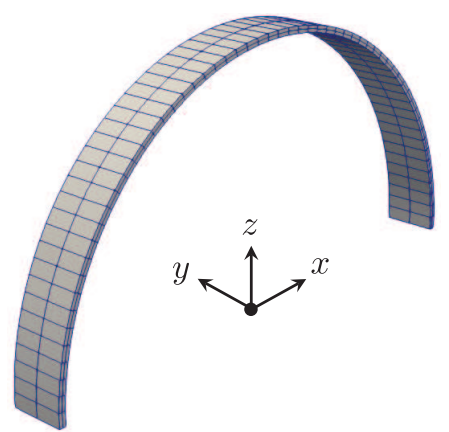

(c)

Figure 9. Meshes of the arch corresponding to (a) $n_{\text {elm }}=10$ elements, (b) $n_{\text {elm }}=20$ elements, and (c) $n_{\text {elm }}=60$ elements along the curvature.

integration of such circuits. Looking at practical issues, more electrical components leads to a greater cost, more parasitic elements, and an increased embedded mass.

\subsection{Electrical analog validation}

3.3.I. Benchmark for the curved beam The considered structure is a fully clamped duraluminum semi-circular arch of radius $R=200 \mathrm{~mm}$, width $b=40 \mathrm{~mm}$, and thickness $h_{s}=5 \mathrm{~mm}$, whose Poisson's ratio $\nu$, density $\rho$, and Young's modulus $Y$ are set at $0.35,2800 \mathrm{~kg} / \mathrm{m}^{3}$, and $69 \mathrm{GPa}$, respectively. Its mode shapes and natural frequencies are estimated via a similar finite element model as in [23]. The structure material exhibits isotropic and homogeneous properties and remains in linear elasticity conditions. It is modeled with 20-node hexahedral elements with one element in depth and two elements in width. Some meshes are illustrated in Figure 9.

The electrical analog of this structure is composed of identical curved beam electrical unit cells, whose lumped-element representation is either sketched in Figure 4 for Model A, or in Figures 6 and 8 for Model B. The structure is discretized with a number of elements $n_{\text {elm }}$ equal to $10,20,30$, or 60 . To tune the network components, the parameters appearing in the frequency coherence conditions (21) have to be estimated.

- The length $a$ depends on the considered discretization: $a=\frac{\pi R}{n_{e l m}}$.

- The discrete mass $m$ is obtained from the mass density: $m=\rho a b h_{s}$.

- The longitudinal stiffness is estimated from $K_{v}=Y \frac{b h_{s}}{a}$.

- The bending stiffness can be computed from $K_{\theta}=Y \frac{b h_{s}^{3}}{12 a}$.

- The main capacitance is given by $C_{\theta}=\frac{150 \cdot 10^{-9}}{n_{e l m} / 10}$.

This means that $C_{\theta}$ is equal to $150 \mathrm{nF}$ for $n_{e l m}=10$, and that it is proportional to the unit cell surface area, which decreases along the curvature when $n_{\text {elm }}$ increases. Even though this numerical example does not involve piezoelectric coupling, this capacitance evolution is inspired from the piezoelectric capacitance dependence on the surface area of the transducers.

- The transformer ratio is arbitrary set at $\hat{a}=4$.

- The electrical radius, the inductance, and the longitudinal capacitance are derived thanks to the frequency coherence conditions in Equation (21):

$$
\hat{R}=\frac{\hat{a} R}{a}, \quad L=\left(\frac{a}{\hat{a}}\right)^{2} \frac{m}{K_{\theta} C_{\theta}}, \quad C_{v}=\frac{m}{L K_{v}} .
$$

In this case, one can prove that $L$ is proportional to $1 / n_{\text {elm }}^{3}$. Thus, there is an interest in increasing the number of unit cells to reduce potential high inductance requirements on the inductors to be produced [21-23]. The values of all these parameters for $n_{\text {elm }}$ from 10 to 60 are recorded in Table 3. 
Table 3. Electrical modeling parameters for different discretizations of the curved beam analog for Model A

\begin{tabular}{|c|c|c|c|c|c|c|c|c|c|}
\hline$n_{\text {elm }}$ & Nodes & $\begin{array}{l}a \\
(\mathrm{~mm})\end{array}$ & $\begin{array}{l}m \\
(\mathrm{~g})\end{array}$ & $\begin{array}{l}K_{v} \\
(\mathrm{GN} / \mathrm{m})\end{array}$ & $\begin{array}{l}K_{\theta} \\
(\mathrm{N} . \mathrm{m})\end{array}$ & $\begin{array}{l}C_{\theta} \\
(\mathrm{nF})\end{array}$ & $\begin{array}{l}\hat{R} \\
(-)\end{array}$ & $\begin{array}{l}L \\
(\mathrm{mH})\end{array}$ & $\begin{array}{l}C_{v} \\
(\mathrm{nF})\end{array}$ \\
\hline 10 & 11 & 62.8 & 35.2 & 0.22 & 458 & 150 & 12.7 & 126.5 & 1.3 \\
\hline 20 & 21 & 31.4 & 17.6 & 0.44 & 915 & 75 & 25.5 & 15.8 & 2.5 \\
\hline 30 & 31 & 20.9 & 11.7 & 0.66 & 1373 & 50 & 38.2 & 4.7 & 3.8 \\
\hline 60 & 61 & 10.5 & 5.9 & 1.3 & 2745 & 25 & 76.4 & 0.6 & 7.6 \\
\hline$n_{\text {elm }}$ & & Meshing & & \multicolumn{3}{|c|}{ MAC matrix } & \multicolumn{3}{|c|}{ Marker } \\
\hline 10 & & Figure 9(a) & & \multicolumn{3}{|c|}{ Figure I I(a) } & \multicolumn{3}{|c|}{ - } \\
\hline 20 & & Figure 9(b) & & \multicolumn{3}{|c|}{ Figure I I(b) } & \multicolumn{3}{|c|}{$\nabla$} \\
\hline 30 & & I & & \multicolumn{3}{|c|}{ Figure II(c) } & \multicolumn{3}{|c|}{ - } \\
\hline 60 & & Figure 9(c) & & \multicolumn{3}{|c|}{ Figure II(d) } & \multicolumn{3}{|c|}{ - } \\
\hline
\end{tabular}

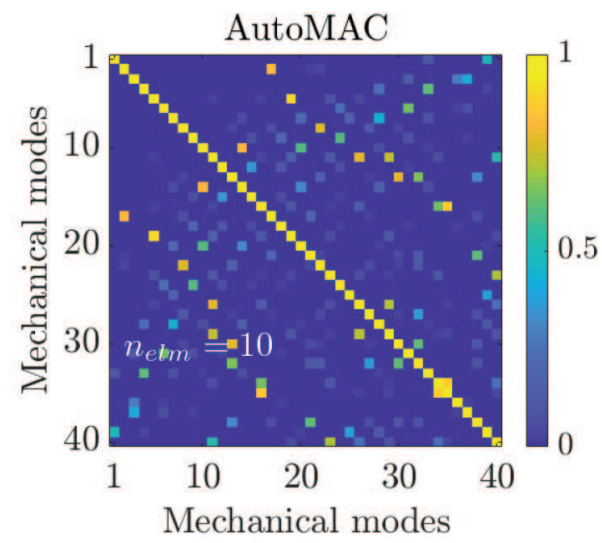

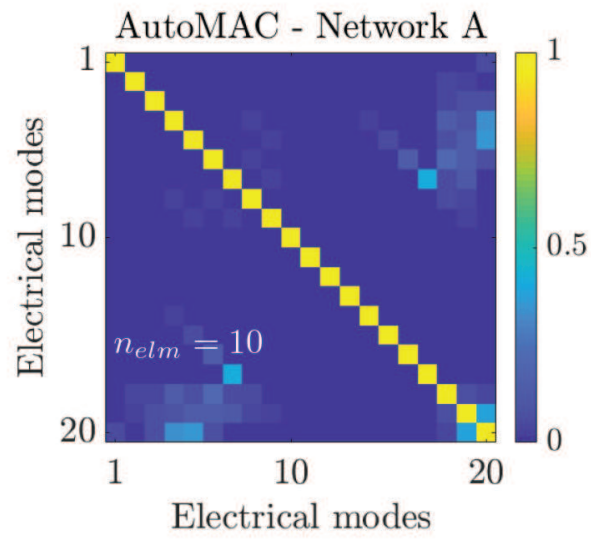

(b)

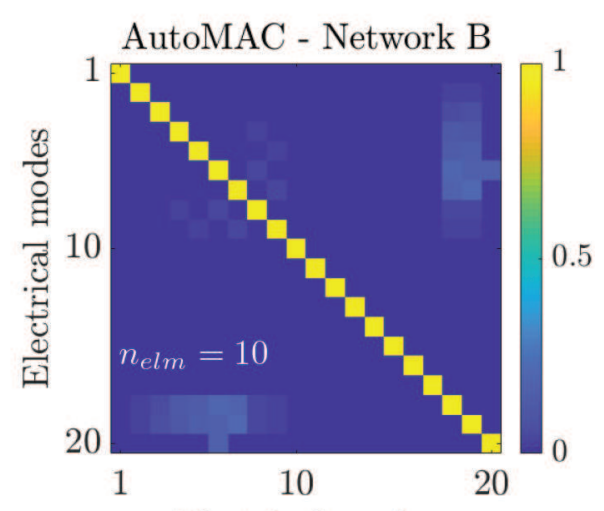

Electrical modes

(c)

Figure 10. (a) AutoMAC of the first 40 velocity modes of the arch, and AutoMAC of the first 20 electrical current modes of the analogous network with (b) Model A and (c) Model B.

3.3.2. Validation of the spatial coherence condition First, we plot the AutoMAC matrices of the mechanical and electrical systems to verify whether the meshes are sufficiently refined. The electrical AutoMAC matrices shown in Figures 10(b) and (c) indicate that all electrical current modes of both networks can be easily distinguished from each other, even for $n_{\text {elm }}=10$. It is thus possible to make the difference between the first 20 electrical modes for a greater number of elements as well. However, the mechanical AutoMAC in Figure 10(a) illustrates a problem of spatial coherence. While it is possible to distinguish most of the 40 first modes of the arch, some are not clearly and uniquely identified. It is the case for example of modes number 2,5 , and 8 , among others. These modes are actually either torsional or out-of-plane bending modes. As written before, and as can be seen 


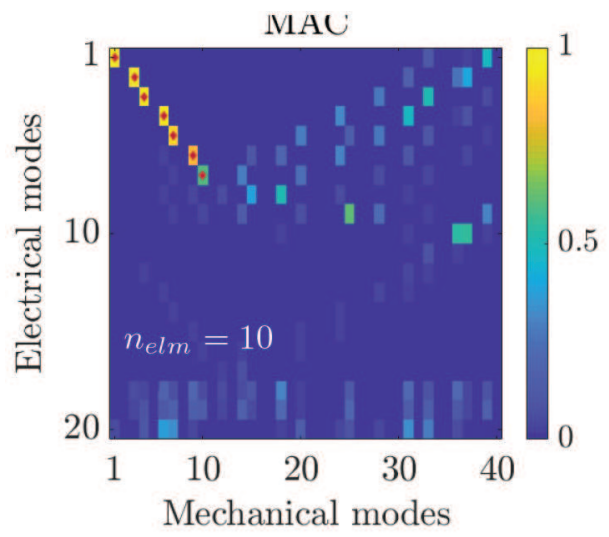

(a)

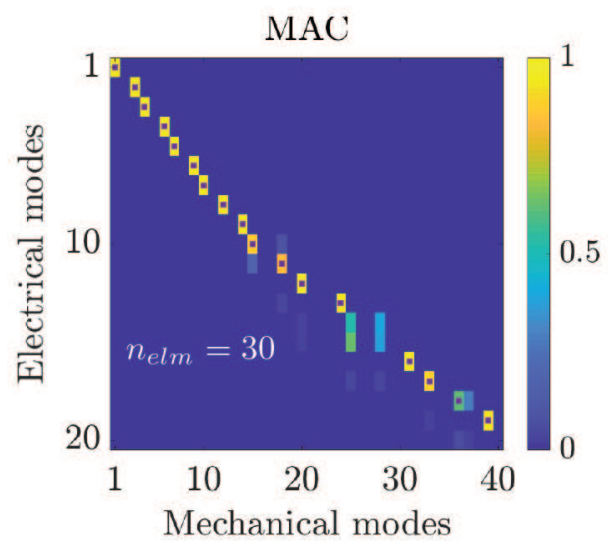

(c)

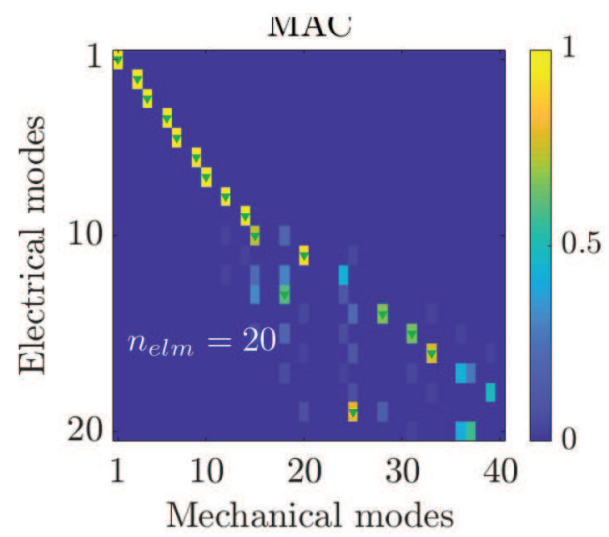

(b)

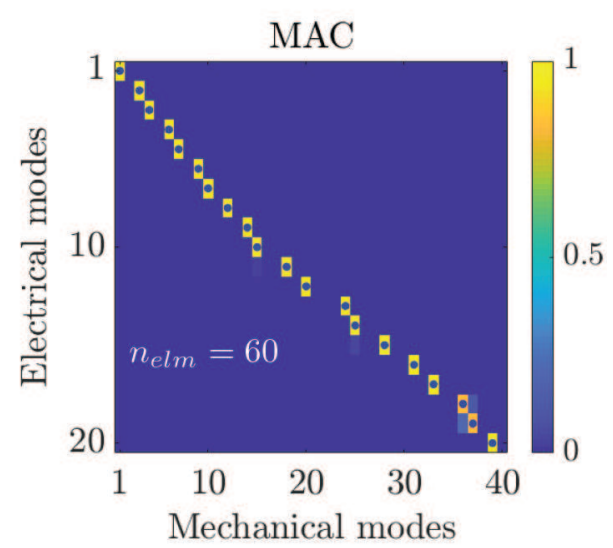

(d)

Figure I I. MAC between the 40 first velocity modes of the arch and the 20 first electrical current modes of the Model A network for (a) $n_{\text {elm }}=10$, (b) $n_{\text {elm }}=20$, (c) $n_{\text {elm }}=30$, and (d) $n_{\text {elm }}=60$. The $\downarrow, \nabla, \mathbf{a}$, and $\bullet$ markers indicate which couple of modes are deemed clearly identified, so that they can be plotted in Figure 12.

in Figure 9, we use only two elements in width and one element in depth. The discretization along these two directions is not fine enough to obtain a completely diagonal AutoMAC matrix, and refining the mesh along the curve would not improve it. Yet, as the focus of the proposed analogs only concerns the in-plane modes, we assume that all mechanical meshes for $n_{\text {elm }} \geq 10$ allow making the difference between mechanical modes we are interested in.

The MAC matrices between electrical current modes of Model A and velocity modes of the curved beam are plotted for different values of $n_{\text {elm }}$ in Figure 11. This is equivalent to comparing arch modes estimated by a finite difference model on one hand, and by a finite element model on the other hand. One can note that the torsional and out-of-plane bending modes of the arch do not appear. Indeed, these kind of loads cannot be predicted by the present network, which only represent in-plane modes. This is why there are some columns of the MAC matrices which completely remain at zero. Note that the results for network B are not shown here but they are very similar which shows that the two networks are equivalent when looking at their first eigenmodes. In both cases, the finite difference models indeed converge towards the finite element model, which is assumed to be precise enough to serve as the reference. Consequently, the spatial coherence condition is met over the first in-plane modes of the arch.

3.3.3. Validation of the frequency coherence condition The natural frequencies of the electrical network are then compared with the arch natural frequencies estimated by a finite element model. We use the mesh presented in Figure 9(c) to ensure computing converged mechanical natural frequencies. The comparison is made in Figure 12 for several numbers of elements $n_{\text {elm }}$. Only the natural frequencies of Model A are plotted. Indeed, the two networks exhibit close natural frequencies: all the natural frequencies of the network representing Model B 


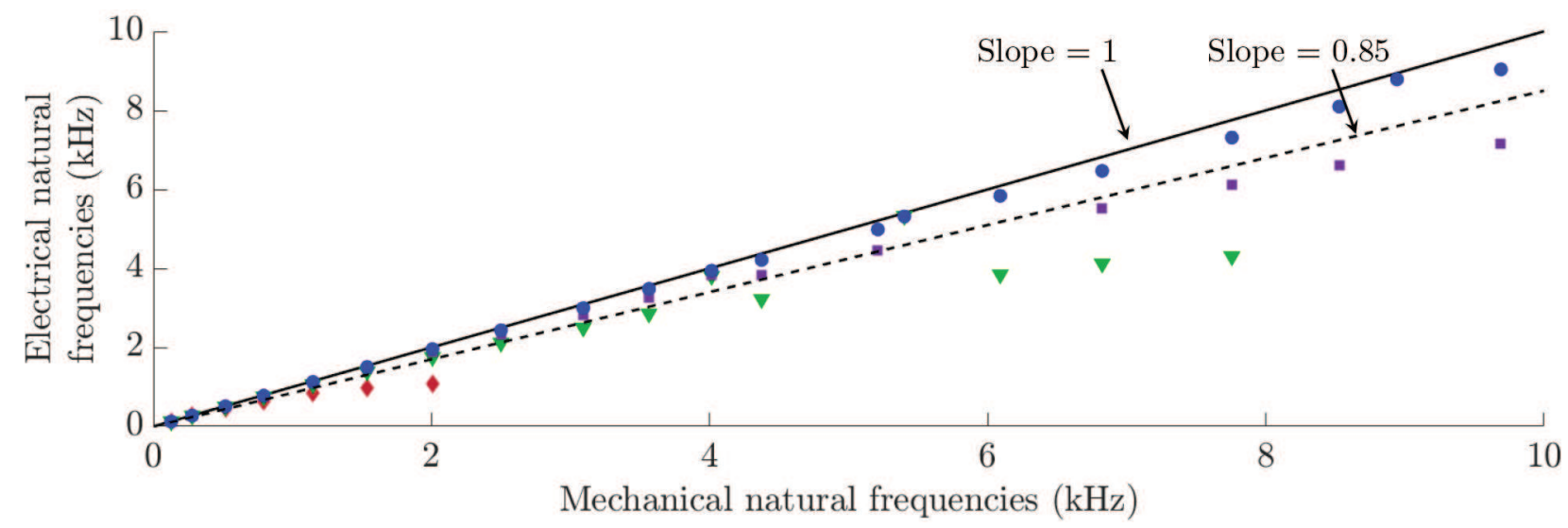

Figure 12. Comparison of mechanical natural frequencies of the arch and electrical natural frequencies of the Model A network for $(\diamond) n_{\text {elm }}=10,(\nabla) n_{\text {elm }}=20,(\square) n_{\text {elm }}=30$, and $(\bullet) n_{\text {elm }}=60$.

are nearly $3.5 \%$ superior to those of Model A for $n_{e l m}=10$ elements, but this gap decreases to less than $1 \%$ for $n_{\text {elm }} \geq 20$ elements. Figure 12 shows that the proposed analog networks converge towards the arch dynamics when the number of elements increases. This can be regarded as the convergence of finite difference models towards a finite element model. Finally, we can say that the frequency range upon which the frequency coherence condition is met depends on the structure discretization. For $n_{\text {elm }}=10$ elements, we can consider that the frequency coherence condition is correctly approximated for the first five in-plane modes. The present numerical example finally shows that the spatial and frequency coherence conditions are validated upon a given frequency range. As expected, increasing the number of elements in the network makes it tend towards a continuous structure, and therefore extends the frequency range over which the analogy is validated.

\section{Piezoelectric network damping of an arch}

\section{I. Electromechanical structure}

A set of piezoelectric patches are bonded to the arch with as much as elements to ensure a sufficient approximation of a continuous electromechanical structure. This means that we consider nearly 10 piezoelectric transducers per wavelength for the mode with the smallest wavelength in the frequency range of interest. The main assumptions for modeling the dynamics of such a coupled system are as follows.

- The infinitesimal strain theory applies.

- The structure is made of duralumin, and behaves as an isotropic homogeneous linear elastic medium. Its Poisson's ratio, density, and Young's modulus are set at $0.35,2800 \mathrm{~kg} / \mathrm{m}^{3}$, and $69 \mathrm{GPa}$, respectively.

- The piezoelectric transducers are thin and polarized in the electrodes orthogonal directions, and they exhibit transverse isotropic properties. They are made of PIC 153 piezoelectric material, whose materials properties are recorded in [23].

- The adhesive layers and the electrodes thicknesses are not modeled.

- The lower electrodes are connected to the ground.

- The electrical network behaves linearly with excitation sources.

- $\quad$ The mechanical damping matrix $D_{m}$ is computed with a hysteretic damping model. The corresponding damping coefficient $\xi$ is set at $0.3 \%$.

From those assumptions, it was shown in $[23,35]$ that the undamped open- and short-circuit dynamics and the static piezoelectric capacitance distribution can be simulated using a finite element model represented by

$$
\left[\left(\begin{array}{cc}
K_{m}+K_{c} K_{e} K_{c}^{\mathrm{T}} & K_{c} K_{e} \\
\left(K_{c} K_{e}\right)^{\mathrm{T}} & K_{e}
\end{array}\right)-\Omega^{2}\left(\begin{array}{cc}
M_{m} & 0 \\
0 & 0
\end{array}\right)\right]\left(\begin{array}{l}
U \\
Q
\end{array}\right)=\left(\begin{array}{l}
F \\
V
\end{array}\right)
$$

The modal properties of the structure with short-circuited patches are estimated using Equation (28) with $V=0$. Meanwhile, the modal properties of the electrical network are directly calculated from the proposed model in 

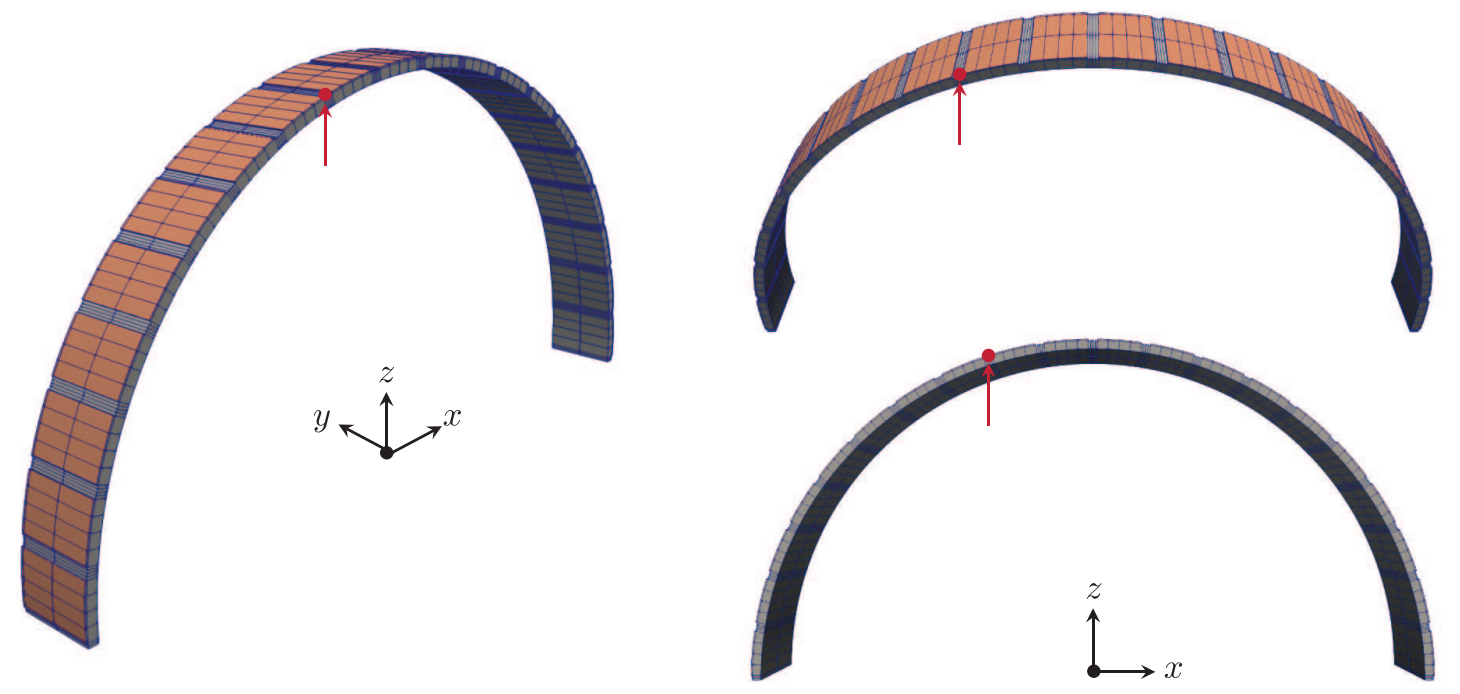

Figure 13. Meshing of the semicircular arch periodically covered by $n=20$ piezoelectric patches, and $\bullet$ location of both the excitation and the velocity measurements for the frequency response functions plotted in Figures 19 and 20.

Equation (25), in which the piezoelectric capacitance replaces the capacitance $C_{\theta}$ of the corresponding unit cell. Note that the electrical unit cells are assembled to form an electrical network with as many cells as there are piezoelectric transducers bonded on the host structure. If both spatial and frequency coherence conditions are met, we consider that the modal coupling condition is met once the structure and its analog network are connected. Note that this is a change of vocabulary from [20-22], where the modal coupling condition expression was used to designate what we now call the frequency coherence condition. Once the modal coupling condition is met, broadband vibration damping is expected if additional resistances are set in the network to adequately add electrical damping. Using the notation of [23], we recall the finite element formulation of a structure being coupled to a lumped electrical network:

$$
\left[\left(\begin{array}{cc}
K_{m}+K_{c} K_{e} K_{c}^{\mathrm{T}} & K_{c} K_{e} P \\
\left(K_{c} K_{e} P\right)^{\mathrm{T}} & K_{N}
\end{array}\right)+\mathrm{j} \Omega\left(\begin{array}{cc}
D_{m} & 0 \\
0 & D_{N}
\end{array}\right)-\Omega^{2}\left(\begin{array}{cc}
M_{m} & 0 \\
0 & M_{N}
\end{array}\right)\right]\left(\begin{array}{c}
U \\
Q_{N}
\end{array}\right)=\left(\begin{array}{c}
F \\
V_{N}
\end{array}\right)
$$

\subsection{Mechanical parameters evaluation}

The semicircular arch represented in Figure 13 is periodically covered with piezoelectric patches of same width $b$ and thickness $h_{p}=h_{s} / 6$ over $5 / 6$ of the external surface of the arch. This means that the length $l_{p}$ of the piezoelectric patches neutral axis is

$$
l_{p}=\frac{5}{6} \frac{\pi\left(R+\frac{h_{s}}{2}+\frac{h_{p}}{2}\right)}{n} .
$$

The radius of the arch is $R=200 \mathrm{~mm}$. Since it is discretized by $n$ elements, $a=\frac{\pi R}{n}$. From the dimensions of the structure, as well as the mass densities of the involved materials, the discrete mass $m$ is

$$
m=\rho a b h_{s}+\rho_{p} l_{p} b h_{p} .
$$

For the stiffness, the discrete mechanical model represented in Figure 14 is considered. A portion of the arch covered by a piezoelectric patch is sketched in the flattened local frame, with $s$ standing for the arc length direction and $w$ denoting the orthogonal direction to $s$ and $y$. Each portion of the element is modeled by a discrete spring. This means that we suggest computing the values of the longitudinal stiffness $K_{v}$ and the bending stiffness $K_{\theta}$ with the following equations:

$$
\frac{1}{K_{v}}=\frac{1}{K_{v 1}}+\frac{1}{K_{v 2}+K_{v 3}}+\frac{1}{K_{v 4}} \quad \text { and } \quad \frac{1}{K_{\theta}}=\frac{1}{K_{\theta 1}}+\frac{1}{K_{\theta 2}+K_{\theta 3}}+\frac{1}{K_{\theta 4}}
$$




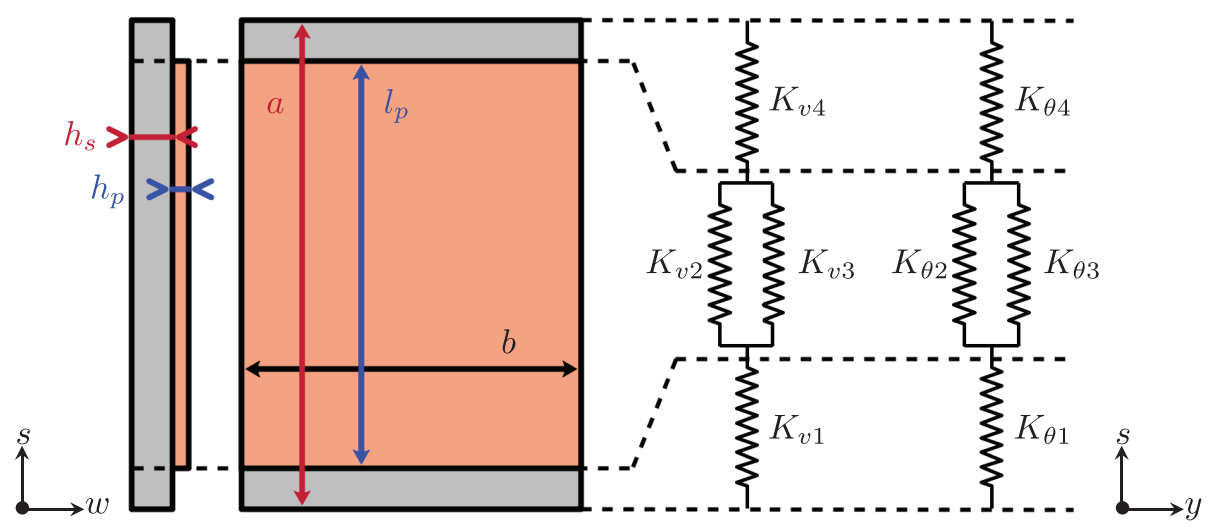

Figure 14. Curved beam element of constant thickness $h_{s}$ covered by a curved piezoelectric patch of constant thickness $h_{p}$. The stiffness of each portion of the beam and the piezoelectric patch can be modeled as a spring, such as drawn on the right hand-side. The represented thicknesses are not to scale.

where the involved discrete stiffnesses, which are indicated in Figure 14, could even be expressed for nonconstant thickness structures through

$$
\begin{aligned}
\frac{1}{K_{v 1}} & =\frac{1}{Y} \int_{0}^{\left(a-l_{p}\right) / 2} \frac{1}{b h_{s}} d s, & \frac{1}{K_{v 3}} & =\frac{1}{1 / s_{11}^{E}} \int_{\left(a-l_{p}\right) / 2}^{\left(a+l_{p}\right) / 2} \frac{1}{b h_{p}} d s, \\
\frac{1}{K_{v 2}} & =\frac{1}{Y} \int_{\left(a-l_{p}\right) / 2}^{\left(a+l_{p}\right) / 2} \frac{1}{b h_{s}} d s, & \frac{1}{K_{v 4}} & =\frac{1}{Y} \int_{\left(a+l_{p}\right) / 2}^{a} \frac{1}{b h_{s}} d s, \\
\frac{1}{K_{\theta 1}} & =\frac{1}{Y} \int_{0}^{\left(a-l_{p}\right) / 2} \frac{1}{I(s)} d s, & \frac{1}{K_{\theta 3}} & =\frac{1}{1 / s_{11}^{E}} \int_{\left(a-l_{p}\right) / 2}^{\left(a+l_{p}\right) / 2} \frac{1}{I_{p}(s)} d s, \\
\frac{1}{K_{\theta 2}} & =\frac{1}{Y} \int_{\left(a-l_{p}\right) / 2}^{\left(a+l_{p}\right) / 2} \frac{1}{I(s)} d s, & \frac{1}{K_{\theta 4}} & =\frac{1}{Y} \int_{\left(a+l_{p}\right) / 2}^{a} \frac{1}{I(s)} d s,
\end{aligned}
$$

where $Y$ and $1 / s_{11}^{E}$ are the Young's modulus of the structure and the short-circuited piezoelectric patches, respectively, $I$ and $I_{p}$ denote the second moments of area of the structure and the piezoelectric patches. In this case, the piezoelectric transducer covering $5 / 6 \approx 83.3 \%$ justifies approximating $I$ and $I_{p}$ with respect to the mid-surface of the entire structure:

$$
I(s)=\int_{0}^{b} \int_{-\left(h_{s}+h_{p}\right) / 2}^{\left(h_{s}-h_{p}\right) / 2} w^{2} d y d w, \text { and } I_{p}(s)=\int_{0}^{b} \int_{\left(h_{s}-h_{p}\right) / 2}^{\left(h_{s}+h_{p}\right) / 2} w^{2} d y d w .
$$

\subsection{Analogous network}

The assembled electrical analog is slightly non-periodic because of the effect of the mechanical boundary conditions on the piezoelectric capacitance. The finite element formulation of Equation (28) can be used to predict the distribution of this static piezoelectric capacitances $C_{s t}$. The distribution, which is plotted in Figure 15(a) for $n=10$, is barely non-periodic because of the mechanical boundary conditions that constrain the structural strain at both ends. In addition, the observed distribution symmetry is consistent with the structure symmetry.

The transformer ratio is arbitrary set at $\hat{a}=4$. The remaining electrical parameters can then be derived by applying the frequency coherence conditions from Equation (21):

$$
\hat{R}=\frac{\hat{a}}{a} R, \quad L=\left(\frac{a}{\hat{a}}\right)^{2} \frac{m}{K_{\theta} C_{s t}}, \quad C_{v}=\frac{m}{K_{v} L} .
$$




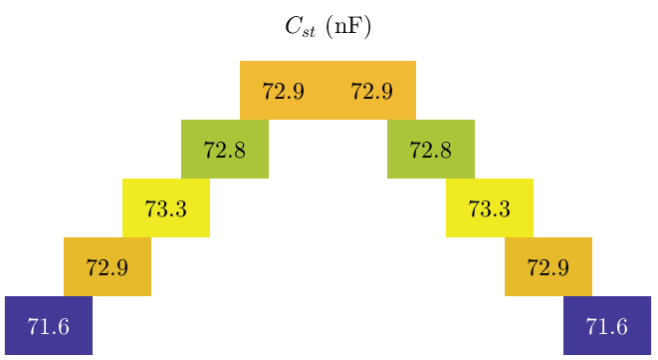

(a)

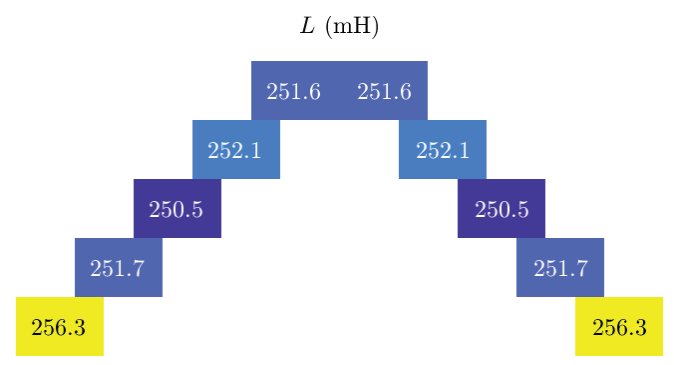

(b)

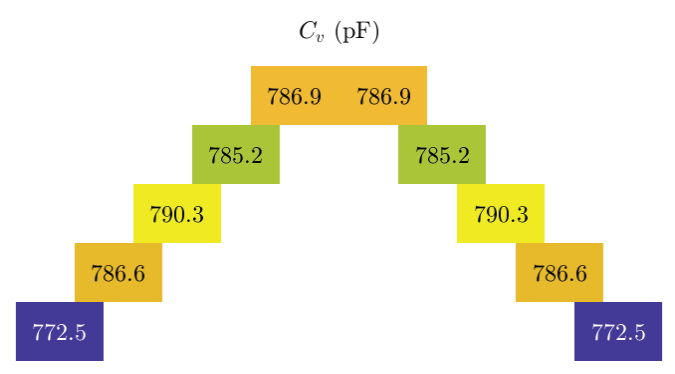

(c)

Figure 15. Distributions of discrete variables over the unit cells for the clamped semicircular arch in the case of $n=10$ : (a) static piezoelectric capacitance $C_{s t}$ (in $\left.\mathrm{nF}\right),(\mathrm{b})$ resulting inductance $L$ (in $\left.\mathrm{mH}\right)$, and (c) resulting capacitance $C_{v}($ in $\mathrm{pF})$.

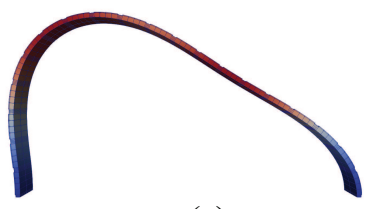

(a)

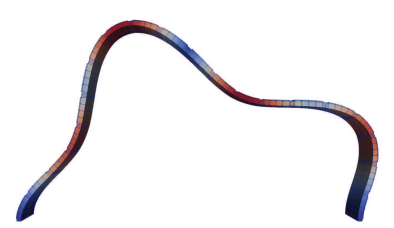

(d)

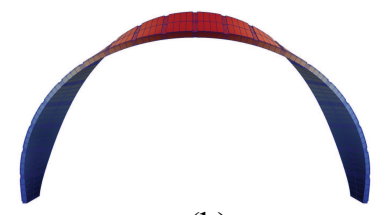

(b)

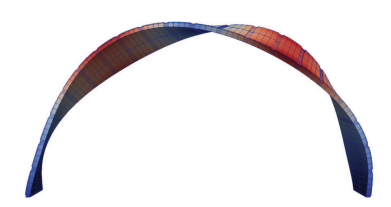

(e)

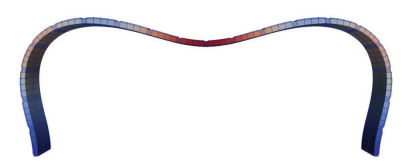

(c)

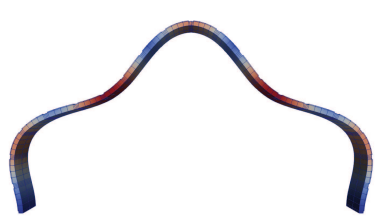

(f)

Figure 16. Shapes of the first six modes and corresponding natural frequencies of the clamped semicircular arch covered with $n=20$ piezoelectric patches: (a) first mode shape at $125.9 \mathrm{~Hz}$; (b) second mode shape at $214.9 \mathrm{~Hz}$; (c) third mode shape at $276.5 \mathrm{~Hz}$; (d) fourth mode shape at $5 \mathrm{I} 3.1 \mathrm{~Hz}$; (e) fifth mode shape at $635.0 \mathrm{~Hz}$; (f) sixth mode shape at $785.6 \mathrm{~Hz}$.

As the static piezoelectric capacitance is the only quantity whose distribution over the arch elements is nonuniform, these equations show that the inductance $L$ distribution is reversed when compared the $C_{s t}$ distribution, whereas the capacitance $C_{v}$ relative distribution is similar to the $C_{s t}$ distribution. Such distributions are represented for $n=10$ in Figures 15(b) and 15(c).

The semicircular arch covered with piezoelectric transducers and the assembled electrical network should exhibit the same modal properties, so that the modal coupling condition is met when they are coupled. Their mode shapes and natural frequencies are simulated using the formulations in Equations (28) and (25), respectively. Looking at the mode shapes of the arch covered with $n=20$ piezoelectric patches, Figure 16 shows that the second and fifth modes of the arch are out-of-plane bending modes, which cannot be reproduced in the electrical network as already shown with the previous purely mechanical arch. Therefore, if they are excited, these modes will appear in the frequency response functions of the structure but they will not be affected by piezoelectric coupling. 


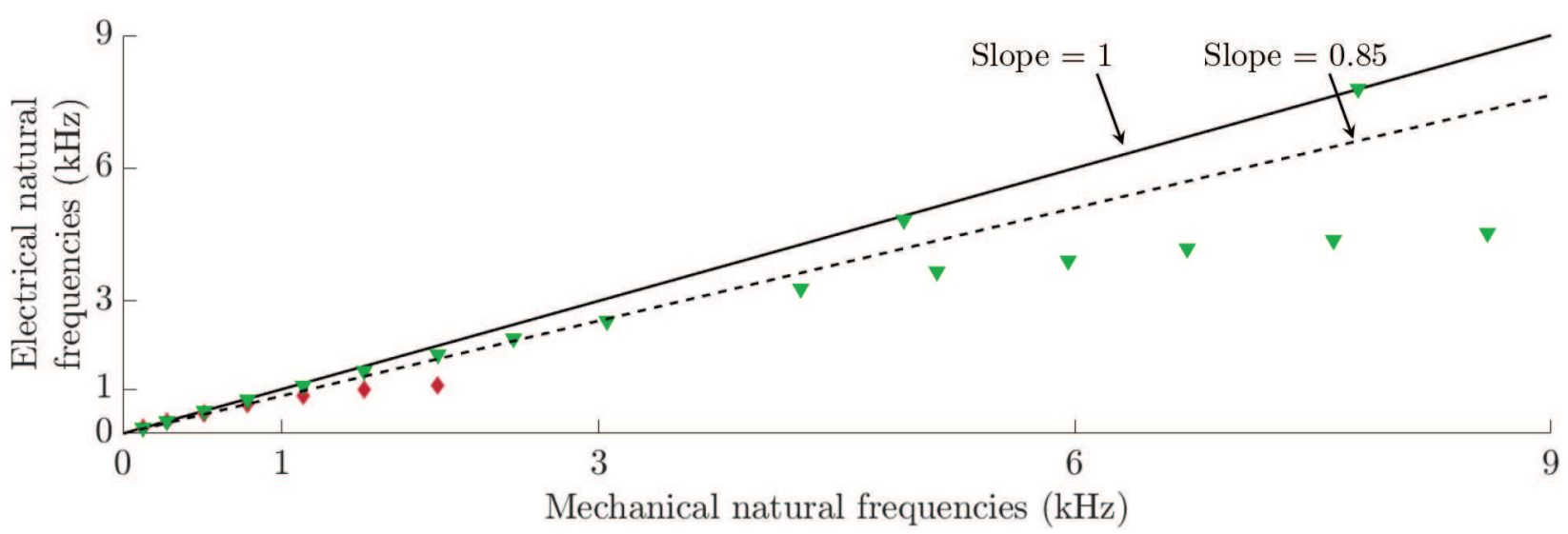

Figure 17. Comparison of mechanical and electrical natural frequencies for $(\diamond) n=10$ and $(\nabla) n=20$.

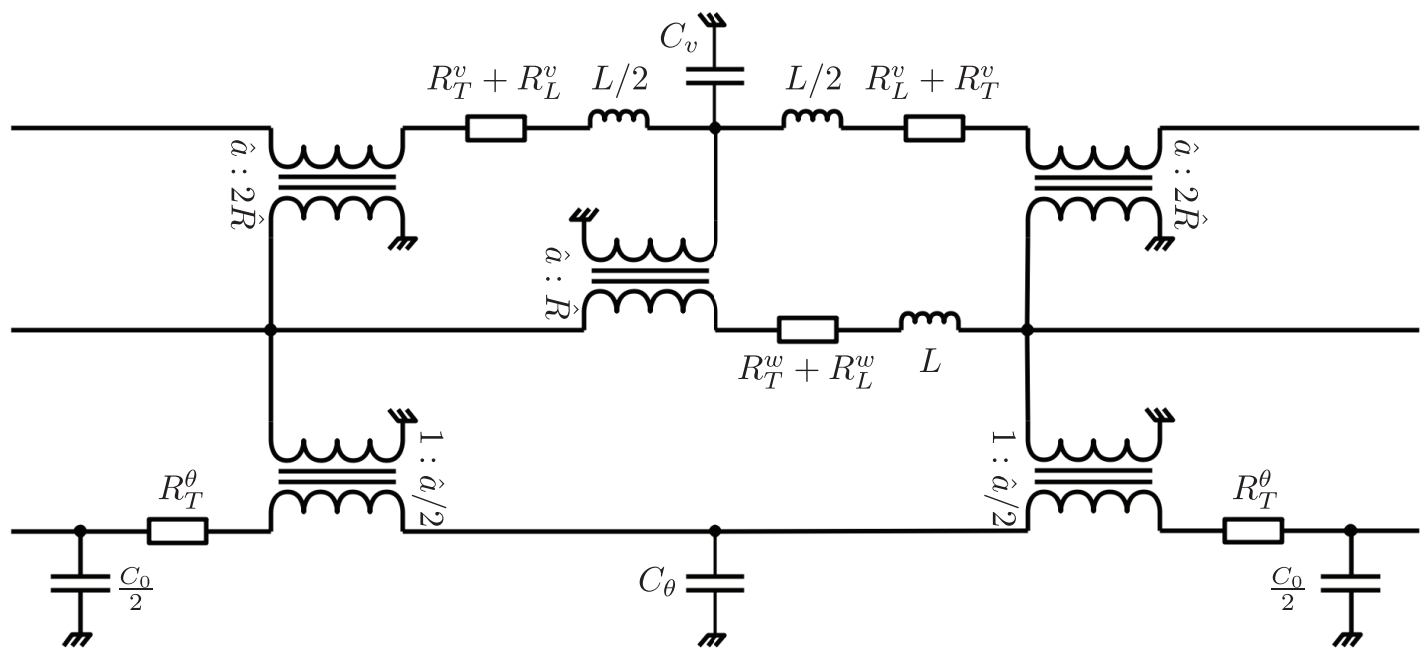

Figure I 8. Unit cell of the dissipative arch electrical analog.

Finally, the natural frequencies of the assembled network are compared to the natural frequencies of the arch in Figure 17. The discussion is quite similar to the previous numerical example without piezoelectric patches: the analogy between the structure and its analog network is ensured over a given frequency range, which extends when increasing the number of elements $n$ discretizing the network. In the present case, one can consider that the analogy is sufficient up to $1 \mathrm{kHz}$ for the first four modes when $n=10$, and up to $3 \mathrm{kHz}$ for the first nine modes when $n=20$.

\subsection{Broadband damping}

Vibration mitigation of the clamped arch covered by piezoelectric patches is achieved by coupling the structure to its analog network. As sketched in Figure 18, dissipative components are included as series resistances for the inductors and transformers of each unit cell. The subscript $T$ stands for the resistance related to transformers, whereas the subscript $L$ stands for the series resistance of inductors. Meanwhile, the superscripts $v, w$, and $\theta$ indicate in which transmission line the components they are associated with are located.

The resistance values are set to $R_{L}^{w}=15 \Omega$ and $R_{L}^{v}=5 \Omega$ for $n=10$ elements, and $R_{L}^{w}=3 \Omega$ and $R_{L}^{v}=1 \Omega$ for $n=20$ elements, which is a realistic estimate considering practical manufacturing considerations [23]. For the transformers, we suggest setting all series resistance $R_{T}^{v}, R_{T}^{w}$, and $R_{T}^{\theta}$ at $10 \Omega$ for $n=10$. It is also a realistic order of magnitude when compared to the transformers produced in [23]. Then, for $n=20$ the value of the inductance $L$ has been divided by around eight, which means that it is reasonable to expect $R_{T}^{v}, R_{T}^{w}$, and $R_{T}^{\theta}$ to be divided by eight as well if they are produced in a similar fashion. 


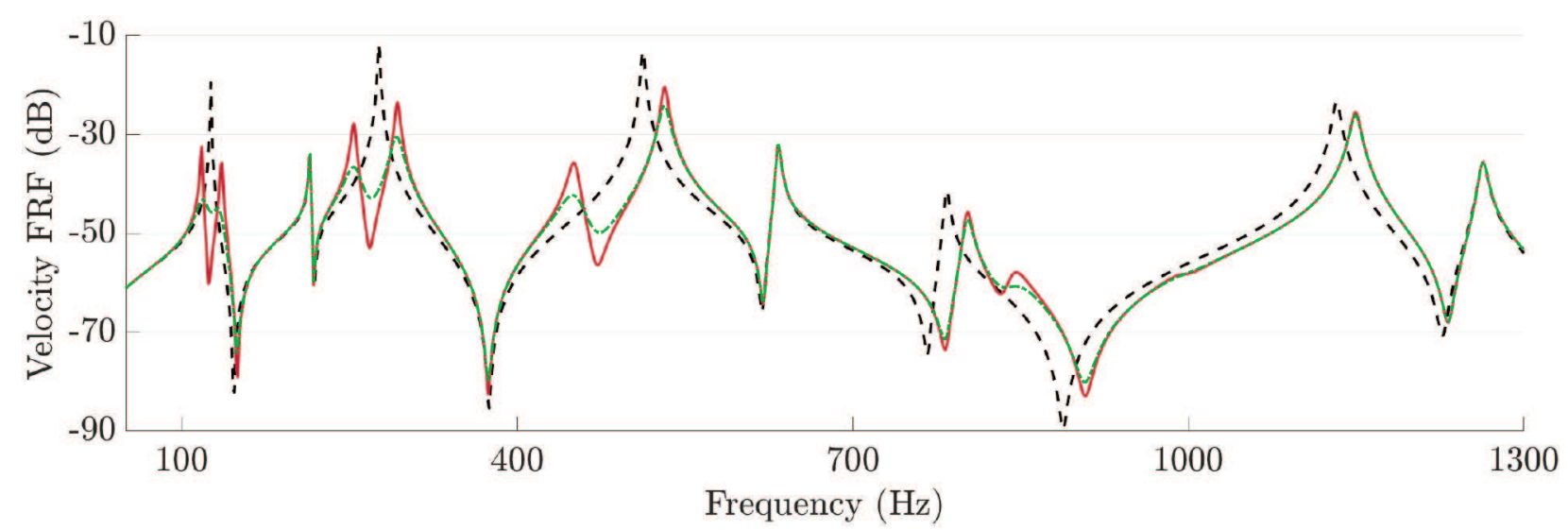

Figure 19. Comparison of simulated velocity frequency response functions for $n=10$ : - - - with short-circuited piezoelectric patches, and when the arch is connected __ to an ideal network, and $-=-$ to a dissipative network.

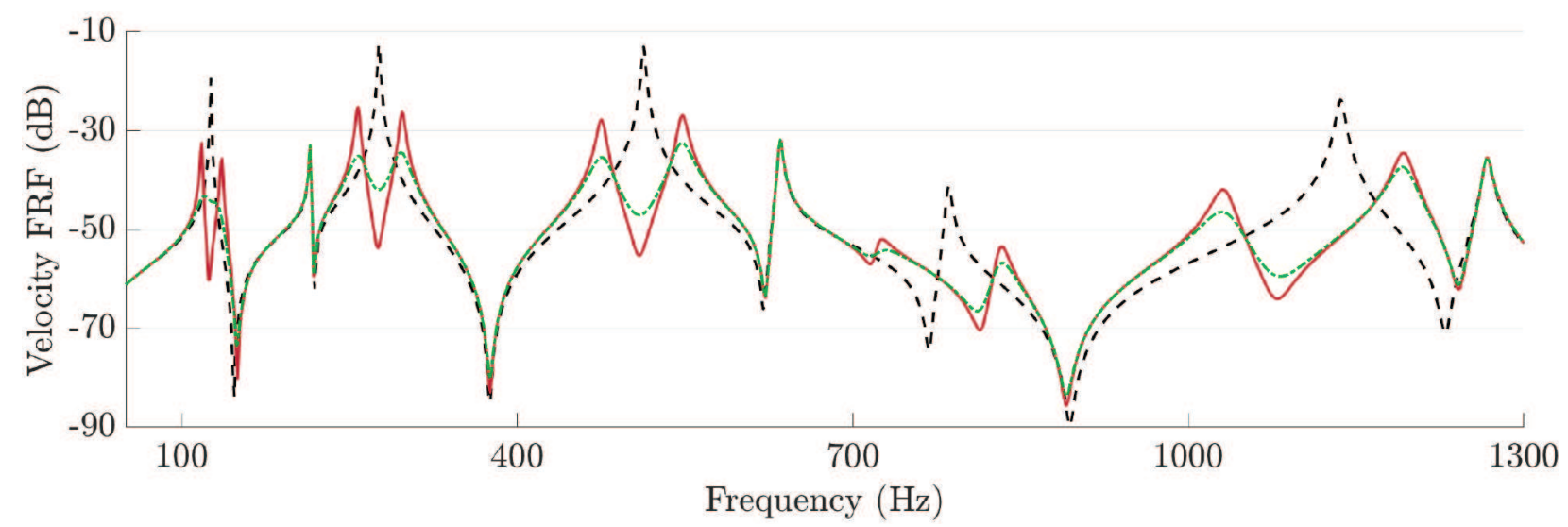

Figure 20. Comparison of simulated velocity frequency response functions for $n=20$ : - - - with short-circuited piezoelectric patches, and when the arch is connected —— to an ideal network and - - - to a dissipative network.

Both the excitation and the measurement are made on the location which is highlighted in Figure 13. This point has been selected in order to excite all modes of the frequency range. In Figures 19 and 20 are plotted the driving-point mobilities obtained from the model of Equation (29). Multimodal damping is achieved over a frequency range that increases when the discretization scheme is refined. The out-of-plane bending modes, which are the second, fifth, and eight modes, are however not affected by the network. This was predictable, based on the fact that these mechanical modes are not represented by electrical modes. Also note that a similar multimodal damping would be achieved by connecting the arch to the analogous network obtained from Model B. Finally, this numerical example is the first demonstration of broadband vibration mitigation of a curved structure coupled to its electrical analog through piezoelectric elements. The proposed electrical components have realistic values, which means that they represent a fully passive broadband damping solution for the semicircular arch.

\section{Conclusions}

In this work a method, similar to those presented in $[2,15,21]$, has been adapted to derive an electrical analog of initially curved beams. The method is based on the application of the direct electromechanical analogy to their Hencky-type discretization as given by Figure 5 [31]. The developed discrete-analog-electrical circuit can replace suitably the continuum model curved beam if two conditions are verified. The first condition is the spatial coherence, which consists of the request that modal shapes reconstructed by using the analog circuit approximate correctly the curved beam mode shapes. The circuit should thus be composed of enough unit cells so that the smaller wavelength of the frequency range of interest is sufficiently discretized. This condition is 
numerically verified by comparing the velocity mode shapes of the curved beam with the electrical current mode shapes of the analog circuit. The second condition is the frequency coherence, which requires that the wave propagation properties calculated for curved beams and their analogs are sufficiently close. Once analogous boundary conditions are obtained for the electrical circuit, it has to be verified that each eigenmode of the curved beam, which belongs to the set of modes to be damped, and its analog electrical eigenmode are sufficiently close.

More specifically, this work has investigated the damping of linear vibrations of curved beams by using the method of multimodal piezoelectric coupling [24]. Here, the first application of this method to a curved beam, and in particular to a semicircular arch, has been presented. The electrical components of the analog circuit are designed in order to ensure the frequency coherence condition. Connecting the curved beam to its analog circuit with dissipative components allows multimodal damping of a fixed set constituted by chosen targeted modes. In order to consider possible prototypal applications, all simulations are conducted with non-

optimized but experimentally feasible components. This means that, also in the considered case, the method of multimodal piezoelectric coupling can produce multimodal damping by using fully passive electric components. It is believed that such a result represents a further step towards the design of more complex piezo-electromechanical structures, eventually aiming to the conception of multi-physics metamaterials [36-40]. To this aim, future works may consider assembling different simple piezo-electro-mechanical microsubstructures in order to synthesize [41-45] novel metamaterials showing an optimized dynamic behavior.

\section{Funding}

The author(s) received no financial support for the research, authorship, and/or publication of this article.

\section{References}

[1] Kron, G. Numerical solution of ordinary and partial differential equations by means of equivalent circuits. $J$ Appl Phys 1945; 16(3): 172-186.

[2] MacNeal, RH. The solution of partial differential equations by means of electrical networks. PhD Thesis, California Institute of Technology, 1949.

[3] MacNeal, RH, McCann, GD, and Wilts, $\mathrm{CH}$. The solution of aeroelastic problems by means of electrical analogies. $J$ Aeronaut Sci 1951; 18(12): 777-789.

[4] Benscoter, SU, and MacNeal, RH. Introduction to electrical-circuit analogies for beam analysis. NACA Technical Note 2785, 1952.

[5] MacNeal, RH. Electrical analogies for stiffened shells with flexible rings. NACA Technical Note 3280, 1954.

[6] Alessandroni, S, dell'Isola, F, and Porfiri, M. A revival of electric analogs for vibrating mechanical systems aimed to their efficient control by PZT actuators. Int J Solids Struct 2002; 39(20): 5295-5324.

[7] Vidoli, S, and dell'Isola, F. Modal coupling in one-dimensional electromechanical structured continua. Acta Mechanica 2000; 141(1-2): 37-50.

[8] Maurini, C, dell'Isola, F, and Del Vescovo, D. Comparison of piezoelectronic networks acting as distributed vibration absorbers. Mech Syst Signal Process 2004; 18(5): 1243-1271.

[9] dell'Isola, F, Maurini, C, and Porfiri, M. Passive damping of beam vibrations through distributed electric networks and piezoelectric transducers: Prototype design and experimental validation. Smart Mater Struct 2004; 13(2): $299-308$.

[10] Bisegna, P, Caruso, G, and Maceri, F. Optimized electric networks for vibration damping of piezoactuated beams. J Sound Vibr 2006; 289(4-5): 908-937.

[11] Pagnini, LC, and Piccardo, G. The three-hinged arch as an example of piezomechanic passive controlled structure. Continuum Mech Thermodyn 2016; 28(5): 1247-1262.

[12] Chróścielewski, J, Schmidt, R, and Eremeyev, VA. Nonlinear finite element modeling of vibration control of plane rod-type structural members with integrated piezoelectric patches. Continuum Mech Thermodyn 2019; 31(1): 147-188.

[13] Porfiri, M, and dell'Isola, F. Multimodal beam vibration damping exploiting PZT transducers and passive distributed circuits. $J$ Physique IV 2004; 115: 323-330.

[14] Giorgio, I, Culla, A, and Del Vescovo, D. Multimode vibration control using several piezoelectric transducers shunted with a multiterminal network. Arch Appl Mech 2009; 79(9): 859-879.

[15] Porfiri, M, dell'Isola, F, and Mascioli, FMF. Circuit analog of a beam and its application to multimodal vibration damping, using piezoelectric transducers. Int J Circuit Theory Applicat 2004; 32(4): 167-198. 
[16] Andreaus, U, dell'Isola, F, and Porfiri, M. Piezoelectric passive distributed controllers for beam flexural vibrations. Modal Anal 2004; 10(5): 625-659.

[17] Porfiri, M, dell'Isola, F, and Santini, E. Modeling and design of passive electric networks interconnecting piezoelectric transducers for distributed vibration control. Int J Appl Electromag Mech 2005; 21(2): 69-87.

[18] Alessandroni, S, Andreaus, U, dell'Isola, F, et al. Piezo-ElectroMechanical (PEM) Kirchhoff-Love plates. Eur J Mech A/Solids 2004; 23(4): 689-702.

[19] Alessandroni, S, Andreaus, U, dell'Isola, F, et al. A passive electric controller for multimodal vibrations of thin plates. Comput Struct 2005; 83(15-16): 1236-1250.

[20] Lossouarn, B, Aucejo, M, and Deü, JF. Multimodal coupling of periodic lattices and application to rod vibration damping with a piezoelectric network. Smart Mater Struct 2015; 24(4): 045018.

[21] Lossouarn, B, Deü, JF, and Aucejo, M. Multimodal vibration damping of a beam with a periodic array of piezoelectric patches connected to a passive electrical network. Smart Mater Struct 2015; 24(11): 115037.

[22] Lossouarn, B, Deü, JF, Aucejo, M, et al. Multimodal vibration damping of a plate by piezoelectric coupling to its analogous electrical network. Smart Mater Struct 2016; 25(11): 115042.

[23] Darleux, R, Lossouarn, B, and Deü, JF. Broadband vibration damping of non-periodic plates by piezoelectric coupling to their electrical analogues. Smart Mater Struct 2020; 29(5): 054001.

[24] Giorgio, I, Galantucci, L, Della Corte, A, et al. Piezo-electromechanical smart materials with distributed arrays of piezoelectric transducers: Current and upcoming applications. Int J Appl Electromag Mech 2015; 47(4): 1051-1084.

[25] Bloch, A. Electromechanical analogies and their use for the analysis of mechanical and electromechanical systems. $J$ Inst Elec Eng I Gen 1945; 92(52): 157-169.

[26] Beranek, LL. Acoustics. Melville, NY: Acoustical Society of America, 1954.

[27] Lossouarn, B, Aucejo, M, Deü, JF, et al. Design of a passive electrical analogue for piezoelectric damping of a plate. J Intell Mater Syst Struct 2017. DOI: 10.1177/1045389x17731232.

[28] Paschero, M. Modeling and synthesis of circuits analogue to generalized ondulatory phenomena giroscopically coupled. PhD Thesis, Sapienza University of Rome, 2007.

[29] Allemang, RJ. The modal assurance criterion - Twenty years of use and abuse. Sound Vibr 2003; 37: 14-23.

[30] Chidamparam, P, and Leissa, AW. Vibrations of planar curved beams, rings, and arches. Appl Mech Rev 1993; 46(9): $467-483$.

[31] Turco, E, dell'Isola, F, Cazzani, A, et al. Hencky-type discrete model for pantographic structures: numerical comparison with second gradient continuum models. Z angew Math Phys 2016; 67(4): 85.

[32] dell'Isola, F, Giorgio, I, Pawlikowski, M, et al. Large deformations of planar extensible beams and pantographic lattices: Heuristic homogenization, experimental and numerical examples of equilibrium. Proc R Soc A Math Phys Eng Sci 2016; 472(2185): 20150790.

[33] Turco, E. Discrete is it enough? The revival of Piola-Hencky keynotes to analyze three-dimensional Elastica. Continuum Mech Thermodyn 2018; 30(5): 1039-1057.

[34] Turco, E, and Barchiesi, E. Equilibrium paths of Hencky pantographic beams in a three-point bending problem. Math Mech Complex Syst 2019; 7(4): 287-310.

[35] Thomas, O, Deü, JF, and Ducarne, J. Vibrations of an elastic structure with shunted piezoelectric patches: Efficient finite element formulation and electromechanical coupling coefficients. Int J Numer Meth Eng 2009; 80(2): 235-268.

[36] Barchiesi, E, Spagnuolo, M, and Placidi, L. Mechanical metamaterials: A state of the art. Math Mech Solids 2019; 24(1): 212234.

[37] dell'Isola, F, Seppecher, P, Alibert, JJ, et al. Pantographic metamaterials: An example of mathematically driven design and of its technological challenges. Continuum Mech Thermodyn 2019; 31(4): 851-884.

[38] Yang, H, Abali, BE, Timofeev, D, et al. Determination of metamaterial parameters by means of a homogenization approach based on asymptotic analysis. Continuum Mech Thermodyn 2019; in press.

[39] Vangelatos, Z, Melissinaki, V, Farsari, M, et al. Intertwined microlattices greatly enhance the performance of mechanical metamaterials. Math Mech Solids 2019; 24(8): 2636-2648.

[40] Spagnuolo, M, and Scerrato, D. The mechanical diode: On the tracks of James Maxwell employing mechanical-electrical analogies in the design of metamaterials. In: Developments and Novel Approaches in Biomechanics and Metamaterials. Berlin: Springer, 2020, pp. 459-469.

[41] Milton, G, Harutyunyan, D, and Briane, M. Towards a complete characterization of the effective elasticity tensors of mixtures of an elastic phase and an almost rigid phase. Math Mech Complex Syst 2017; 5(1): 95-113.

[42] Milton, G, Briane, M, and Harutyunyan, D. On the possible effective elasticity tensors of 2-dimensional and 3-dimensional printed materials. Math Mech Complex Syst 2017; 5(1): 41-94.

[43] Barchiesi, E, dell'Isola, F, and Hild, F. On the validation of homogenized modeling for bi-pantographic metamaterials via digital image correlation. Int J Solids Struct 2020; in press.

[44] Spagnuolo, M, Yildizdag, ME, Andreaus, U, et al. Are higher-gradient models also capable of predicting mechanical behavior in the case of wide-knit pantographic structures? Math Mech Solids 2021; 26(1): 18-29.

[45] Yildizdag, ME, Tran, CA, Barchiesi, E, et al. A multi-disciplinary approach for mechanical metamaterial synthesis: A hierarchical modular multiscale cellular structure paradigm. In: State of the Art and Future trends in Material Modeling. Berlin: Springer, 2019, pp. 485-505. 


\section{Appendix: Elementary matrices for the curved beam electrical analog}

The derivation of the curved beam electrical analog elementary matrices is explained in Section 3.1. The matrix $B$ is invertible as long as $C_{0} \neq 0$, in which case the dynamic matrix $D_{e}$ can be computed. Equation (11) is then applied. We recall the non-dimensionalized parameters which appear in the following expressions:

$$
\delta=\frac{\hat{a}}{\hat{R}}, \quad \beta=L C_{v} \Omega^{2}, \quad \gamma=\hat{a}^{2} L C_{\theta} \Omega^{2}, \quad \epsilon=\frac{C_{0}}{C_{\theta}} .
$$

As a consequence, the elementary matrices corresponding to the unit cell of the curved beam electrical analog in Figure 4 are

$$
\begin{aligned}
& M_{e l m}=\frac{L}{4}\left(\begin{array}{cccccc}
2+\frac{\delta^{2}}{4} & \frac{\delta}{2} & \frac{\hat{a} \delta}{4} & -\frac{\delta^{2}}{4} & \frac{\delta}{2} & -\frac{\hat{a} \delta}{4} \\
\frac{\delta}{2} & 1 & \frac{\hat{a}}{2} & -\frac{\delta}{2} & 1 & -\frac{\hat{a}}{2} \\
\frac{\hat{a} \delta}{4} & \frac{\hat{a}}{2} & \frac{\hat{a}^{2}}{4} & -\frac{\hat{a} \delta}{4} & \frac{\hat{a}}{2} & -\frac{\hat{a}^{2}}{4} \\
-\frac{\delta^{2}}{4} & -\frac{\delta}{2} & -\frac{\hat{a} \delta}{4} & 2+\frac{\delta^{2}}{4} & -\frac{\delta}{2} & \frac{\hat{a} \delta}{4} \\
\frac{\delta}{2} & 1 & \frac{\hat{a}}{2} & -\frac{\delta}{2} & 1 & -\frac{\hat{a}}{2} \\
-\frac{\hat{a} \delta}{4} & -\frac{\hat{a}}{2} & -\frac{\hat{a}^{2}}{4} & \frac{\hat{a} \delta}{4} & -\frac{\hat{a}}{2} & \frac{\hat{a}^{2}}{4}
\end{array}\right), \\
& K_{\text {elm }}=\frac{1}{\hat{a}^{2} C_{0}} \frac{1}{\epsilon \gamma \delta^{2}+16 \beta(\epsilon+1)} K_{0},
\end{aligned}
$$

where $K_{0}$ is a $6 \times 6$ symmetric matrix, whose terms above the diagonal are

$$
\begin{aligned}
& K_{0}^{(1)}=\left(\begin{array}{c}
16 \epsilon \gamma+16 \beta \delta^{2}+16 \epsilon^{2} \gamma-8 \delta^{2} \epsilon^{2} \gamma+\delta^{4} \epsilon^{2} \gamma+16 \beta \delta^{2} \epsilon-8 \delta^{2} \epsilon \gamma+2 \delta^{4} \epsilon \gamma \\
*
\end{array}\right), \\
& K_{0}{ }^{(2)}=\left(\begin{array}{c}
2 \delta\left(16 \beta+16 \beta \epsilon-4 \epsilon \gamma-4 \epsilon^{2} \gamma+\delta^{2} \epsilon^{2} \gamma+2 \delta^{2} \epsilon \gamma\right) \\
4 \gamma \delta^{2} \epsilon^{2}+8 \gamma \delta^{2} \epsilon+64 \beta \epsilon+64 \beta \\
*
\end{array}\right), \\
& K_{0}^{(3)}=\left(\begin{array}{c}
2 \hat{a} \delta\left(\epsilon \gamma \delta^{2}+8 \beta+8 \beta \epsilon-2 \epsilon \gamma\right) \\
4 \hat{a}\left(\epsilon \gamma \delta^{2}+8 \beta+8 \beta \epsilon\right) \\
2 \hat{a}^{2}\left(\epsilon \gamma \delta^{2}+8 \beta+16 \beta \epsilon\right) \\
*
\end{array}\right) \\
& K_{0}^{(4)}=\left(\begin{array}{c}
16 \beta \delta^{2}-16 \epsilon \gamma-16 \epsilon^{2} \gamma+8 \delta^{2} \epsilon^{2} \gamma-\delta^{4} \epsilon^{2} \gamma+16 \beta \delta^{2} \epsilon+8 \delta^{2} \epsilon \gamma \\
2 \delta\left(16 \beta+16 \beta \epsilon+4 \epsilon \gamma+4 \epsilon^{2} \gamma-\delta^{2} \epsilon^{2} \gamma\right) \\
4 \hat{a} \delta(4 \beta+4 \beta \epsilon+\epsilon \gamma) \\
16 \epsilon \gamma+16 \beta \delta^{2}+16 \epsilon^{2} \gamma-8 \delta^{2} \epsilon^{2} \gamma+\delta^{4} \epsilon^{2} \gamma+16 \beta \delta^{2} \epsilon-8 \delta^{2} \epsilon \gamma+2 \delta^{4} \epsilon \gamma \\
*
\end{array}\right)
\end{aligned}
$$




$$
\begin{gathered}
K_{0}^{(5)}=\left(\begin{array}{c}
-2 \delta\left(16 \beta+16 \beta \epsilon+4 \epsilon \gamma+4 \epsilon^{2} \gamma-\delta^{2} \epsilon^{2} \gamma\right) \\
4 \gamma \delta^{2} \epsilon^{2}-64 \beta \epsilon-64 \beta \\
-32 \hat{a} \beta(\epsilon+1) \\
-2 \delta\left(16 \beta+16 \beta \epsilon-4 \epsilon \gamma-4 \epsilon^{2} \gamma+\delta^{2} \epsilon^{2} \gamma+2 \delta^{2} \epsilon \gamma\right) \\
4 \gamma \delta^{2} \epsilon^{2}+8 \gamma \delta^{2} \epsilon+64 \beta \epsilon+64 \beta \\
*
\end{array}\right), \\
K_{0}^{(6)}=\left(\begin{array}{c}
4 \hat{a} \delta(4 \beta+4 \beta \epsilon+\epsilon \gamma) \\
32 \hat{a} \beta(\epsilon+1) \\
16 \hat{a}^{2} \beta \\
2 \hat{a} \delta\left(\epsilon \gamma \delta^{2}+8 \beta+8 \beta \epsilon-2 \epsilon \gamma\right) \\
-4 \hat{a}\left(\epsilon \gamma \delta^{2}+8 \beta+8 \beta \epsilon\right) \\
2 \hat{a}^{2}\left(\epsilon \gamma \delta^{2}+8 \beta+16 \beta \epsilon\right)
\end{array}\right) .
\end{gathered}
$$

